Portland State University

PDXScholar

$11-3-2006$

\title{
An Examination of Conflict Conversation in an Online Community: the Pie Fight at DailyKos.com
}

Samantha Isabella Soma

Portland State University

Follow this and additional works at: https://pdxscholar.library.pdx.edu/open_access_etds

Part of the Communication Technology and New Media Commons, Peace and Conflict Studies Commons, and the Social Control, Law, Crime, and Deviance Commons Let us know how access to this document benefits you.

\section{Recommended Citation}

Soma, Samantha Isabella, "An Examination of Conflict Conversation in an Online Community: the Pie Fight at DailyKos.com" (2006). Dissertations and Theses. Paper 1466.

https://doi.org/10.15760/etd.1465

This Thesis is brought to you for free and open access. It has been accepted for inclusion in Dissertations and Theses by an authorized administrator of PDXScholar. Please contact us if we can make this document more accessible: pdxscholar@pdx.edu. 


\section{THESIS APPROVAL}

The abstract and thesis of Samantha Isabella Soma for the Master of Science in Conflict Resolution were presented November 3, 2006, and accepted by the thesis committee and the department.

COMMITTEE APPROVALS:

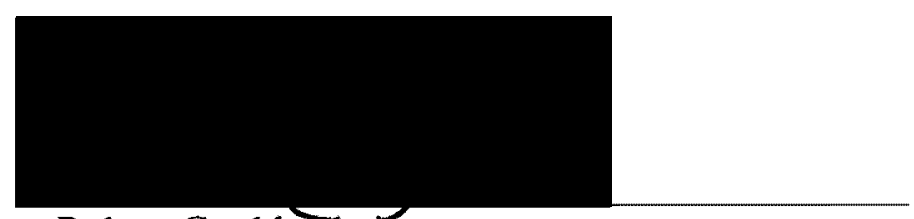

Robert Gould, Chair

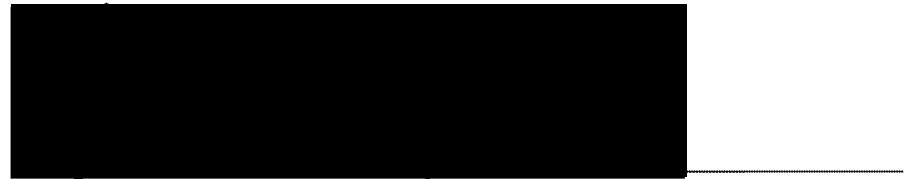

Amanda Byron

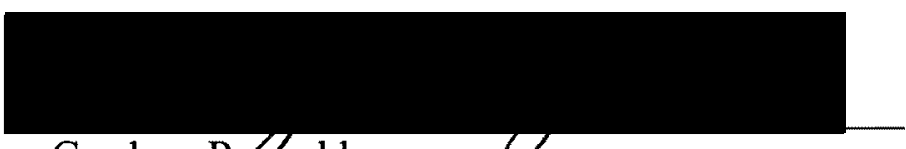

Candyce Refholds

C

Representative of the Office of Graduate Studies

DEPARTMENT APPROVAL:

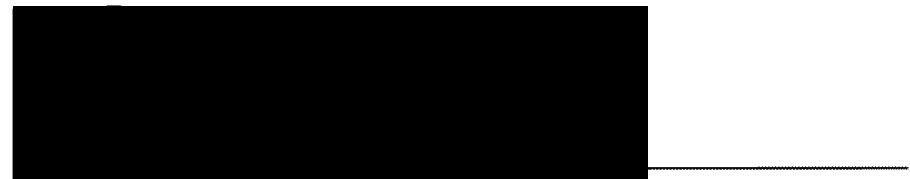

Robert Gould, Wrector

Conflict Resolution Program 


\begin{abstract}
An abstract of the thesis of Samantha Isabella Soma for the Master of Science in Conflict Resolution presented November 3, 2006.
\end{abstract}

Title: An examination of conflict conversation in an online community: the Pie Fight at DailyKos.com

The increasing popularity of the social web and online communities requires the attention of researchers of conflict resolution. Although there are many ways to resolve conflict offline, the ways in which conflict takes place within a native online context have not been much studied. Are any of the tools and strategies that are used to improve communication offline used successfully online, or are some other strategies taking their place? What communication patterns occur within an online community equipped with comment moderation capabilities? This research is a case study and addressed these questions by performing a qualitative analysis of comment conversations within two diaries that discussed a conflict event known as the Pie Fight within the Daily Kos community in June, 2005.

The findings of this research are organized into three sections, which discuss behaviors related to Communication Style, Conflict Minimization or Avoidance, and community members' response to Comment Moderation (Ratings). Novel communication style behaviors which were noted included the use of cut-and-paste 
"paraphrasing" which was used to escalate conflict rather than resolving it, medium blaming when the writer's own words were quite obviously provocative, and extended leave-taking as a means of maintaining relationships before departing from the community. Conflict minimization or avoidance behaviors included the assumption that removal of discussion about the conflict by deleting an entire diary and comments threads would somehow resolve the disagreements included in them it, the use of benign verbal aggression which seemed to bring some segments of the community together, and the temporary or permanent departure from the community by members who took part in the conflict discussion. Ratings moderation created another point of conflict when negative ratings were threatened, although negative ratings were used mainly to address unproductive language rather than punish differences of opinion. The use of positive ratings to offer silent support or appreciation to members whose reasoned comments received a dismissive response was also observed. These findings could be used in future research about the suitability of online communities as sites of deliberative discussion. 
'AN EXAMINATION OF CONFLICT CONVERSATION

IN AN ONLINE COMMUNITY:

THE PIE FIGHT AT DAIL YKOS.COM

by

SAMANTHA ISABELLA ŞOMA

A thesis submitted in partial fulfillment of the requirements for the degree of

\author{
MASTER OF SCIENCE \\ in \\ CONFLICT RESOLUTION
}

Portland State University

2007 


\section{ACKNOWLEDGMENTS}

My chair, program director Robert Gould, was the first person I met in the Conflict Resolution program, and from the beginning I have been impressed by his warmth, integrity, and compassion as he has guided and accompanied me through various stages of this academic journey. His unfailing support and behind the scenes perspective have been appreciated more than I have conveyed.

Candyce Reynolds has been a consistently benevolent presence throughout my time at Portland State, and has encouraged me through many different, wideranging thesis ideas. From her, I've learned how to be a more engaged learner and engaging facilitator, and her mentorship is one of the reasons that I have gone on to pursue a Ph.D.

Amanda Byron undertook her responsibilities as my second reader with grace and skill, and great insight about my process. Her generosity and her ability to ask pointed questions about what I was saying, and what I wasn't, has improved the quality of this work.

I would like to thank the University Studies Department and the Center for Academic Excellence for their support, intellectual and financial, while I've been at Portland State. In addition to making the completion of this degree possible, my teaching and research assistantships have greatly enriched my skill set, and enlarged my circle of friends. 
From the Conflict Resolution program, thank you to Barbara Tint, Kathleen Herron, Clarice Bailey, Mary Zinkin, Suzy Driver, Les Swanson, Stephenie Jahnke, and Nancy Weaver for the care and attention that you gave to me and to each of the Conflict Resolution students who've passed through your doors.

A special thank you to Isbel Ingham, for the wisdom, compassion, and activism she has modeled and bestowed upon me and others, and to Brandy Parris, for her unwavering friendship, perceptiveness, and love through the years.

My parents, Phil and Averil, and my older brothers Cas and Phil, have been my exemplars as I go through life, and they have helped shape the person that I've become. I'm grateful to them for more reasons than I can articulate here. Much gratitude also, to my family-in-law-their interested questions and continued encouragement were appreciated more than I ever said.

While I could have done this without Bill, I'm so grateful that I never had to. He's been a rock of support in all the ways that matter, in this and all other things, and continues to make the journey meaningful and worthwhile. 


\section{TABLE OF CONTENTS}

Acknowledgments ............................................................................................................

List of Figures ...........................................................................................................................vi

Chapter 1 - Introduction ................................................................................................

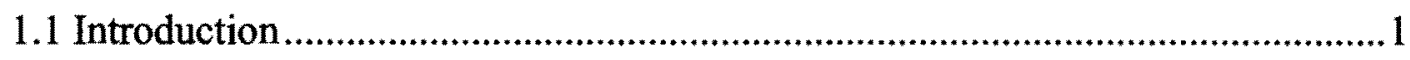

1.2 From Blog to Community - A Brief History of Online Discussion Groups .........3

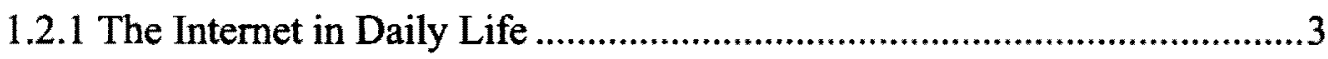

1.2.2 From Individual to Community Blogs .....................................................

1.2.3 From Targeted to Open Discussions.........................................................

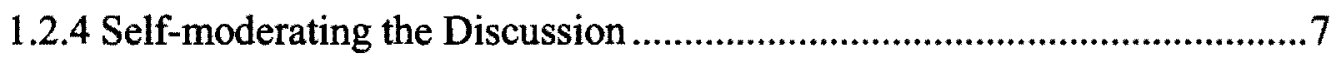

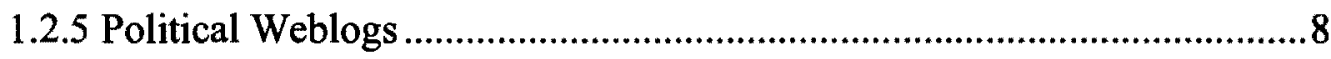

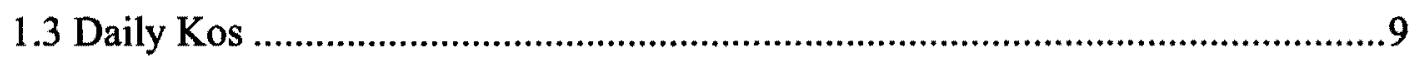

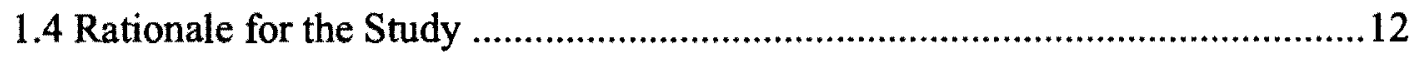

Chapter 2 - Review of the Literature ............................................................................14

2.1 Conflict and Interpersonal Communication .....................................................14

2.2 Computer Mediated Communication versus Face to Face Communication.......17

2.3 Participation and Rules Conduct within Online Discussion Groups ..................18

2.4 Self-expression and Conflict Online ..............................................................19

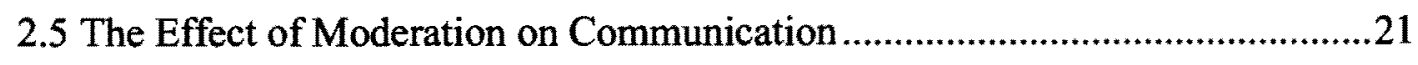

2.6 Conflict within Online Communities ...............................................................21 


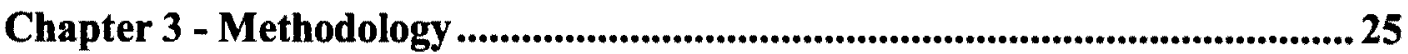

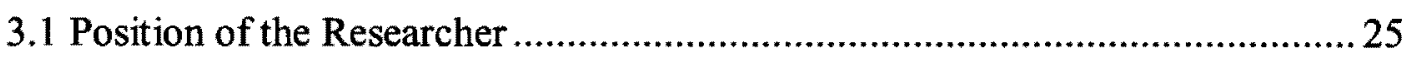

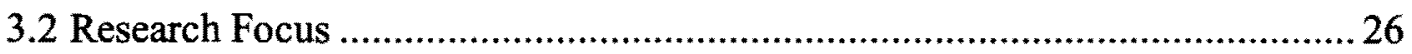

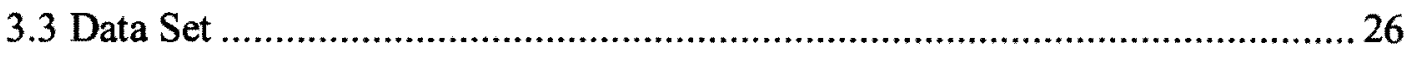

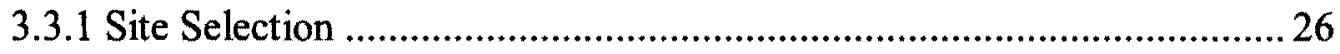

3.3.2 Description of Pie Fight Incident ....................................................... 27

3.3.3 Why this Case?

3.3.4 Total Number of Authors, Comments, Ratings …................................. 29

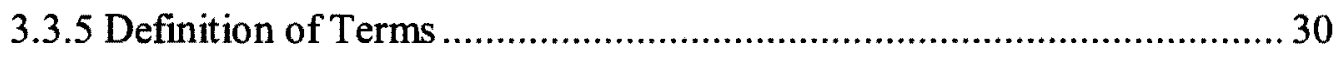

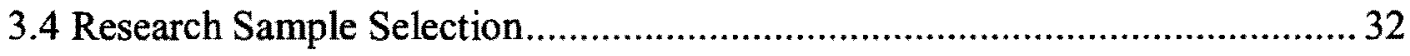

3.4.1 Comments Threads and Conversation Threads ...................................... 32

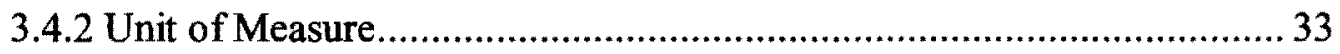

3.4.3 Limitations - Hidden Comments ............................................................ 34

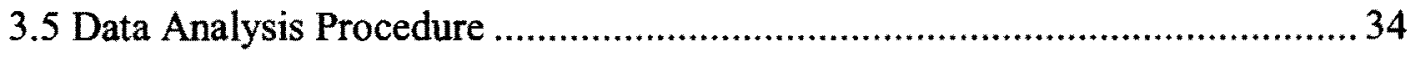

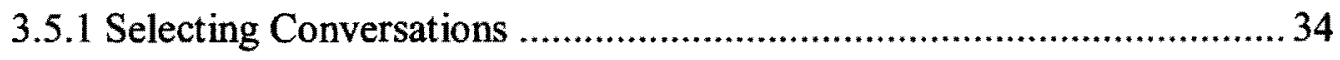

3.5.2 Thematic Content Coding ................................................................... 36

Chapter 4 - Analysis and Discussion ..................................................................3

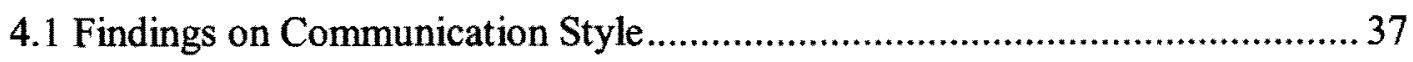

4.1.1 Finding 1 - Quoting, not Paraphrasing .................................................... 37

4.1.2 Finding 2 - Apologizing ...................................................................... 40

4.1.3 Finding 3 - Using "I" or "You"................................................................. 42 


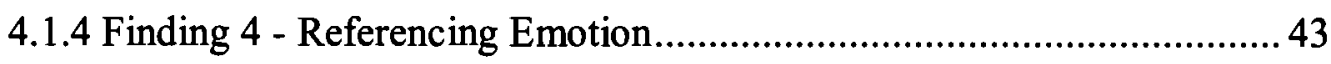

4.1.5 Finding 5 - Switching Personalities .................................................... 43

4.1.6 Finding 6 - Extended Leave-taking ........................................................ 46

4.2 Findings on Conflict Minimization or Avoidance ........................................... 46

4.2.1 Finding 7 - Minimizing Conflict.............................................................. 46

4.2.2 Finding 8 - Deleting Content.............................................................. 48

4.2.3 Finding 9 - Threatening to Depart, Taking a Breather ........................... 50

4.2.4 Finding 10 - Departing Permanently ................................................... 51

4.2.5 Finding 11 - "Invitation" to Exit ........................................................... 52

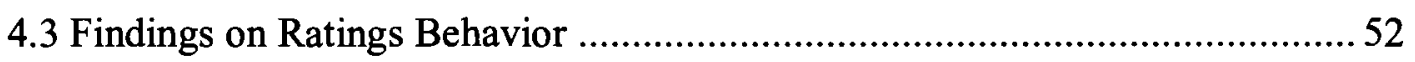

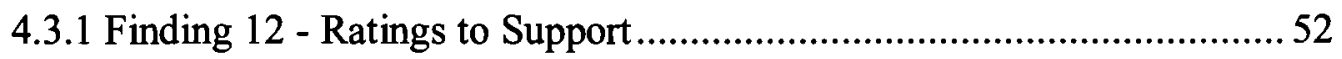

4.3.2 Finding 13 - Ratings to Threaten ........................................................ 53

4.3.3 Finding 14 - Ratings to Punish................................................................ 54

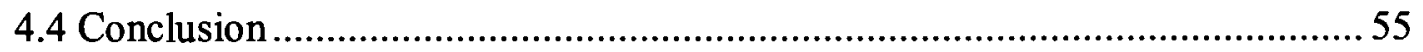

Chapter 5 - Limitations and Future Research ........................................................... 56

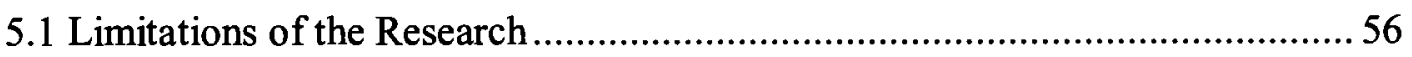

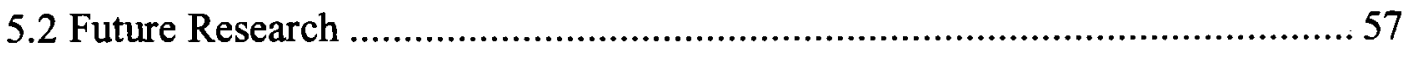

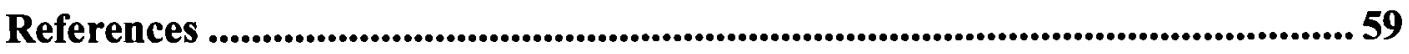

Appendix A - Diaries and Conversation Threads .................................................... 63

Appendix B - Additional Background on the Daily Kos Site................................65 


\section{LIST OF FIGURES}

Figure 1 - Front page of the Daily Kos website ......................................................10

Figure 2 - Ratings Description .............................................................................. 11 


\section{CHAPTER 1 - INTRODUCTION}

\subsection{Introduction}

The Internet is rapidly becoming the communication medium of choice for an increasing number of Americans. A 2003 Pew Internet and American Life Project report claimed that online communities were increasing in scope (Horrigan 2003), and that people were going online to supplement or augment their offline communities (Boase, Horrigan, Wellman, and Rainie 2006). Time Magazine just named Internet site YouTube as its 2006 Invention of the Year (Grossman 2006). The technology which drives YouTube is seen as an exemplar of the newly emerging "social web", also known as Web 2.0 , which relies on community members' cocreation of the content of the site, and of its community (Madden and Fox 2006). People turn to these online communities to fulfill professional and social needs, such as the creation of professional social networks (http://www.LinkedIn.com), spirited discussion of television shows (http:/televisionwithoutpity.com), "due diligence" research and information on political candidates and issues (http://www.mydd.com), and the sharing of photos (http://www.flickr.com) or videos (http://www.youtube.com).

As more people go online, they will be exposed to different methods and means of communication, and an increasingly diverse group of people. Early electronic communities had either few methods with which to deal with or mitigate conflict (Usenet, particularly), or they relied on expulsion of disruptive members, and in later cases, barriers to entry such as requiring users to register before being 
allowed to participate in the community. This led to the belief that electronic communities are either places of high conflict or of limited diversity of views (Davis 1999). In the first case, this limited their usefulness for those who do not work well within these constraints. In the latter case, it limited their value to only those people who would work well within such a constraint. Given the increasing popularity of electronic communities, it seems that recent technical developments have improved community functionality, which increases the likelihood that electronic communities can support the discussion and resolution of a diversity of views.

However, there are lingering concerns about whether or not computer mediated communication (CMC) can support complex or conflict laden discussions because of the lack of interpersonal cues that are such a large part of face-to-face communication. Conflict resolution typically requires communicating an active awareness of both one's own and the other's interests throughout the course of a conflict (Wilmot and Hocker 1998). Are the tools and strategies that we rely on to mitigate conflict off-line, such as reflective listening or paraphrasing, able to be used online, or are some other strategies taking their place? This research attempts to answer that question by undertaking the examination of a conflict that occurred within an established online community. 


\subsection{From Blog to Community - A Brief History of Online Discussion Groups}

\subsubsection{The Internet in Daily Life}

In 2003, only a small segment of the online population reported regularly reading blogs ("The Blogging Iceberg" 2003). Today, however there is little question of their relevance to the millions of readers who go online for purposes that are independent of their own communication needs (Rainie and Horrigan 2005). The growth patterns of the Internet and blog use show no significant signs of slowing, and indeed if disposable income for other entertainment or media use diminishes, access to the Internet becomes even more important (Rainie and Horrigan 2005).

The prospects of the Internet as a tool for increased deliberation have been discussed a great deal prior to and in the wake of both the 2000 and 2004 U.S. Presidential elections. However, if online venues become just another area where people of like mind merely reinforce one another's views, as has been feared by their detractors, this promise becomes muted, if not nonexistent.

Interestingly enough, it is the fact that computer mediated communication (CMC) offers no physical conversational cues (which has been denigrated in earlier research on online communication) that might make this diverse communication possible. Although McKenna and Green (2002) and others have demonstrated that the social effects of what happens in virtual communications are no less real than those which occur offline in face to face interactions, CMC remains an important venue for those who might otherwise be limited by the social "gating" functions that 
govern face to face interactions and which hinder those who might not pass through the gate.

\subsubsection{From Individual to Community Blogs}

In July 1999, the first free build-your-own-web page creation software, pitas, was made available; it was followed by Blogger one month later (Blood 2002). Both tools enabled users to easily create their own web pages, included a mechanism for 'pasting' links and provided a layout, so that creating and posting web pages became as simple as using a word processor. The era of web logs (blogs) encouraged more web surfing as bloggers sought to find the most unique and/or newsworthy links to share with their readership, shifting the focus of the publisher and the user from news and information about the individual site owner outwards, toward the web at large. Online publishing was transformed from a personal journaling venue to a one-tomany content delivery stream, with site owners publishing links and commentary with an increasing awareness of their audience.

The "blogging revolution" engendered a great deal of publicity and discussion about the purposes and uses of personal blogs. Were they merely an enhanced version of Usenet, a means by which individuals could connect with other "weirdoes" like themselves, or were they actually the means by which true online communities could be formed? Beginning in 1999, numerous news articles hailed the advent of this new web medium, and the number of blogs has increased exponentially every year since. In 2003 , the estimate was that there would be over ten million hosted blogs by the end of 2004 ("The Blogging Iceberg " 2003). In 
2005, the Pew Internet and American Life project found that more than 8 million people have created a blog, and that 32 million people regularly read blogs (Rainie 2005).

Community blogs, following on the heels of Usenet discussion groups, allowed for multiple posting of links and commentary to a single blog. Slashdot ${ }^{1}$ and MetaFilter $^{2}$ are the best known early examples of this format, although there are now innumerable different community blog (also known as filter) sites. Community blogs or filters give publication power to any member of the community, broadening the field of discourse to as many as would like to participate. Of course, not all of the members in filter blogs post links, or even comment, but this "free rider" issue is of much less import when there is such a large but anonymous contingent of community members (or merely readers). The "free rider" problem of nonparticipators (also known as lurkers) taking from the community while giving nothing back, is less of an issue in the Internet space than in a face-to-face venue-online lurkers are invisible, and while they do not participate in the gift culture itself, they do not necessarily diminish it by their non-communication either (Preece 2001).

\subsubsection{From Targeted to Open Discussions}

Discussion subjects on MetaFilter run the gamut from politics to pop culture to personal problems, and are only limited by the efforts of the community members

\footnotetext{
${ }^{1}$ http://www.slashdot.org

${ }^{2} \mathrm{http}: / /$ www.metafilter.com
} 
to find interesting web site links to add to the discussion. Usenet lists are organized by topic (such as alt.politics.party, alt.soc.abortion, alt.tv.lost) which screens out any "not relevant" content--in MetaFilter, any topic posted by a community member is considered relevant to its user base. This self-policing aspect of group weblogs is yet another difference between earlier researched online communities.

In MetaFilter and other community blogs, posted links are seldom deleted, although the community members might register their disagreement or disgust with those links that are personally offensive. The MetaFilter site also has a separate area in which to discuss the discussions that take place on the site. MetaTalk ${ }^{3}$ allows users to discuss posting etiquette and other questions or comments about the site "offline" from the threaded discussions that take place on the main page. The ability to discuss the state of the community adds another level of interactivity to the site - rather than grouse about issues "in secret" the discussions about the health of the community take place where those interested can participate in the discussion, but those who do not care can opt out.

The MetaFilter group blog site is owned by an individual who hosts the site, controls who is able to participate and who, on rare occasion, will remove unrepentant, obstructive users from the community ${ }^{4}$. This capability to discuss

\footnotetext{
${ }^{3} \mathrm{http}: / / \mathrm{www}$. metatalk.com

${ }^{4}$ See the thread concerning the banning of "Rightwinger" by site owner "mathowie" here: http://metatalk.metafilter.com/mefi/419 \#2915 for discussion of a rare instance in which a user was banned from the MetaFilter community.
} 
discussions within the same site is what makes blogs different from previously studied online communities, and more similar to the self-reflective discussions which occur on some newspapers' public editor columns wherein the editor critiques or responds to reader critiques of the newspaper's reportage.

\subsubsection{Self-moderating the Discussion}

Next in the community blog's evolution was the advent of user moderation and judgment of comment value, first seen on the Slashdot ${ }^{5}$ technology community blog, which focuses on "News for Nerds. Stuff that matters." The moderation system enabled all participating members of the community to have the opportunity to moderate and rate individual comments; community members who consistently post comments that were deemed worthwhile by the membership were rewarded with higher "karma points" and may receive community privileges based on these ratings (Powazek 2002; CmdrTaco 2003). For example, as designated "trusted users," their own comment ratings might be given greater importance and weight, and their opinions more often solicited by other community members, than those of someone with fewer karma points might. The only limitation is that a user may not post on a comment thread in which they are participating. Because Slashdot and MetaFilter are privately owned and run, the site owners are the final arbiters of what is allowed on their sites, but banning people is often a last resort, only occurring after other methods of conflict resolution have been attempted (Haughey 2002). Other thematic

\footnotetext{
${ }^{5}$ http://www.slashdot.org
} 
community blogs followed MetaFilter's lead, and then the practice spread to individually owned blogs, which began to include the ability for readers to comment on the blog owner's posts, creating the possibility for communities to form around a single person's site.

\subsubsection{Political Weblogs}

Over time, blogs have formed around specific topics. One common topic focused on political discussions, often from a particular political leaning. Participants can write an opinion or link to a current news story, and then other participants can comment and build a discussion.

Comments-enhanced political blogs combine the media aspects of early media blogs with the community aspect of community filter blogs. The majority of these blogs offer their readers a space to post comments either signed or anonymous, but with no formalized registration procedure aside from a text recognition test to prevent spamming of the comments area. In the more sophisticated political weblogs, site owners may require users to register with a unique ID or user name before they may comment. Some sites also allow for the arrangement of comments chronologically by thread topic, instead of only chronologically (which might increase the likelihood that users will respond to each other instead of merely spouting off indiscriminately), and even allow users to rate each others' comments, which is intended to increase the depth and breadth of participation within the community. Although there is always the possibility that only comments with which the rater agrees will receive a high rating, this capability also allows readers to ignore 
comments rated below a certain threshold. This is the same problem as the echo chamber issue mentioned by (Sunstein 2001), but it also permits a more focused exploration of the commentary within a site.

\subsection{Daily Kos}

The political weblog in which this research takes place is Daily $\operatorname{Kos}^{6}$ (See Figure 1). The site has existed since May 26, 2002, has grown from 5,000 registered members and around 3.1 million site visits per month in early 2004 (Markos Moulitsas Zúniga, personal communication, February 11, 2004), to the current statistics of almost 80,000 registered users and about 12 million site visits a month (Sitemeter 2006).

Daily Kos (so named because "Kos" was the owner's nickname when he served in the U.S. Army) is owned by Markos Moulitsas Zúniga, who operates the site full time and is supported by ample advertising revenue. User registration is required for those who would like to comment on the site, although non-registered users may read every area of the site (with the exception of hidden comments accessible only to Trusted Users). While previous iterations of the site (and many other political weblogs) did not employ this barrier to participation, Daily Kos moved to this format in October of 2003. The reasons for this change included the fact that the anonymous comment format incurred several cases of trolling ${ }^{7}$,

\footnotetext{
${ }^{6}$ http://www.dailykos.com

${ }^{7}$ A troll is "a newsgroup post that is deliberately incorrect, intended to provoke readers, or a person who makes such a post." From: http:/www.archivemag.co.uk/glory/T.html
} 
mistaken identity, and even identity theft. As well, the inability of a user to read comments in any way but chronologically, or to reply directly to a comment that occurred earlier in the thread, hindered the interactivity of the site and the substance of the commentary. This does not mean that users did not quote other users to designate to what or to whom specifically they were replying, but the process required an extra step that not every user chose (or was technologically astute enough) to employ. It is interesting to note that this "shortcoming" remains on Eschaton, which is currently the second most trafficked comments-enabled political weblog (Sitemeter 2006).

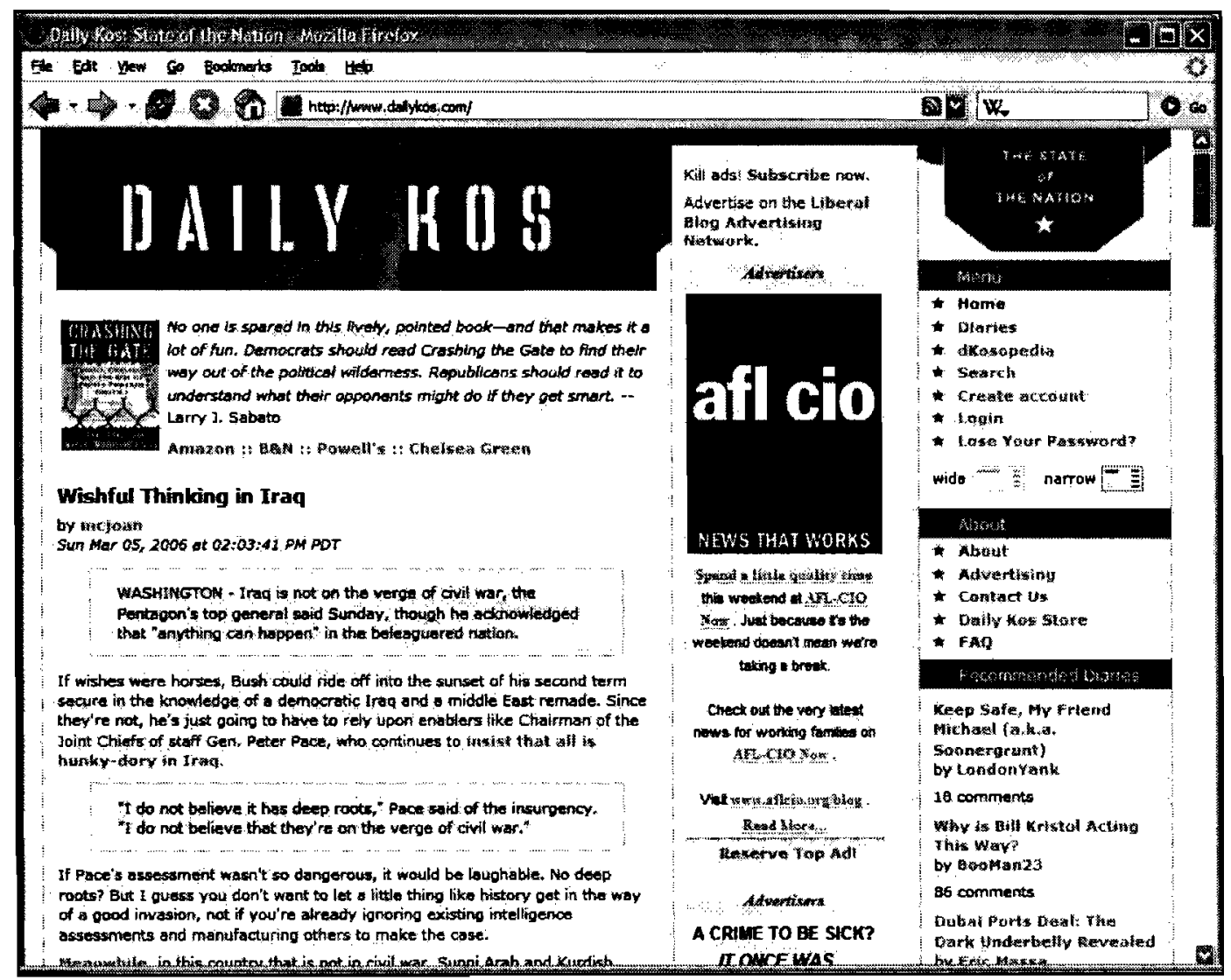

Figure 1 - Front page of the Daily Kos website. 
Figure 1 shows the main elements of the Daily Kos site. Front Page stories are to the left, advertisements are in the middle column, and to the right are the user controls for the site. The Menu, on the top right, contains direct links to the homepage, Diaries, dKosopedia (which is an information page about the site), a Search engine, Create account page, Login for members who have created an account, and an auto generated email if a user has lost their password. Just below the Main menu is the About section of the site, and below that is the list of Recommended Diaries. Not shown on this image, just below the Recommended Diaries list, is the list of Recent Diaries, which refreshes every time a new diary is posted.

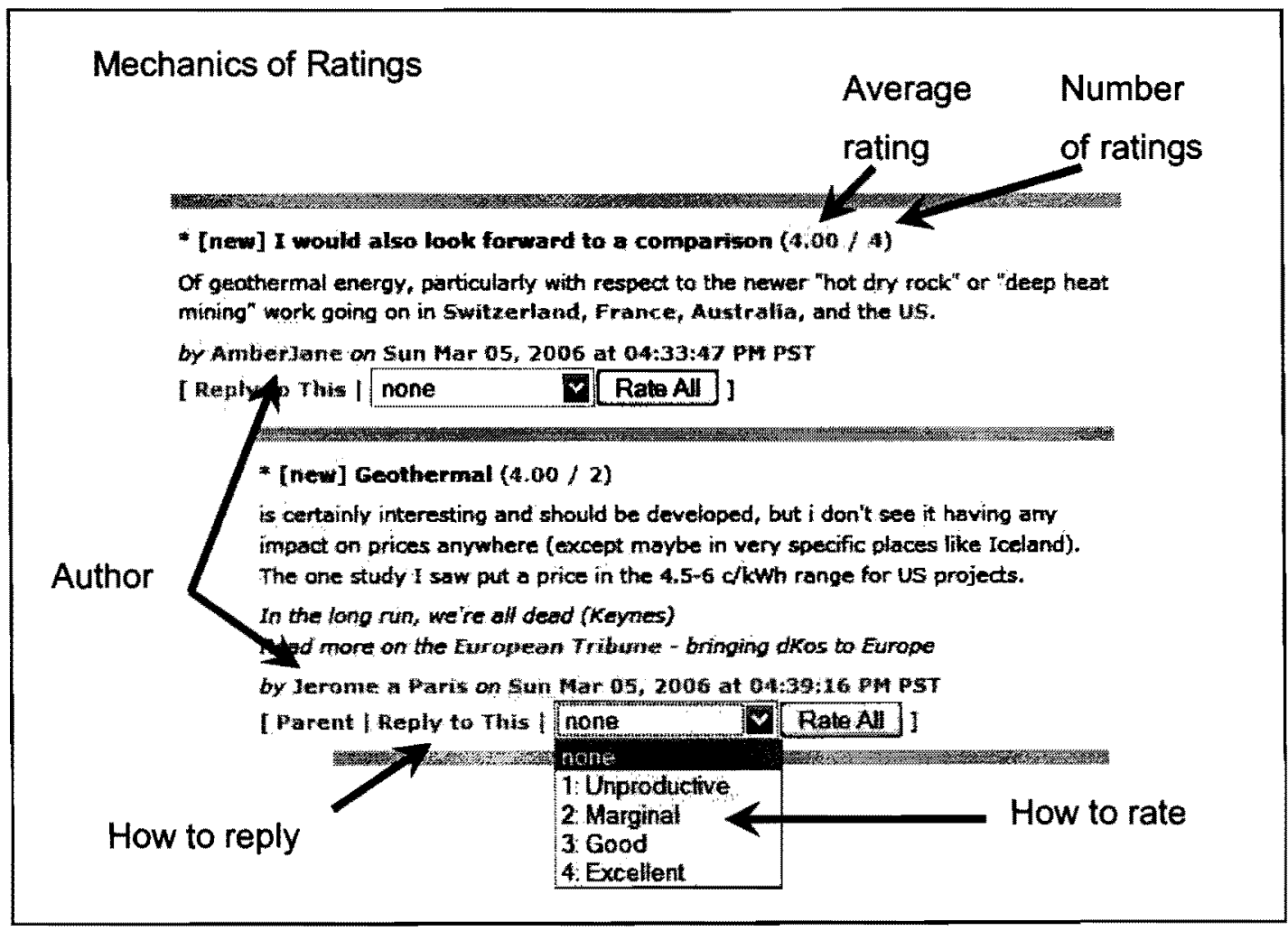

Figure 2 - Ratings Description 
Figure 2 shows the key features involved in commenting or rating on the Daily Kos site, including the Reply to This link, and the Ratings dropdown menu, which is only shown to members who are logged in to the site. The gray bars separate comments from each other. The indentations give a visual indication of the comment that is being responded to. Gray bars which that share the same left margin indicate that the comments below are responding to a single comment above them on the page. In this figure, the position of the second and third bars indicate that the comments below are part of a single thread, with each comment responding to the one directly above it. ${ }^{8}$

\subsection{Rationale for the Study}

While people typically go online to meet needs that cannot be met offline, these needs are different for each person. A person who can discuss political issues with many like-minded others offline is more likely to go online to seek different examinations of the issue. Similarly, a person who might hold his tongue in the offline world, where his views are in the minority, would be more likely to seek to connect with people who share his viewpoints. As more people move online to discuss issues, they will want tools that enhance deliberation, such as more sophisticated interfaces that assist the reader in digesting or dismissing certain types of content. A comment ratings system can also help those people who do not have a

\footnotetext{
${ }^{8}$ As of March 9, 2006, the ratings system of the site has been changed to a simple $+/-$ system, with only trusted users allowed to give minuses. A potential area of future research is to study whether this change has had any effect on the number or types of comments that are posted.
} 
lot of extra time to screen out the commentary that has been judged as less relevant or valuable by their community members. However, it is important to recognize the danger that Sunstein (2001) might consider is inherent in this type of moderationthat users will merely use the ratings system to homogenize the debate rather than valuing the comments for their contribution no matter what their actual position on the discussion.

This research takes the first step in determining whether or not this homogenizing behavior occurs within the Daily Kos site, by examining how conflict plays out in the community. It differs from earlier research on political discussion groups, which looked at the way people fight about differing, deeply held political beliefs (Davis 1999; Sunstein 2001). In this case, the community under study is seen by its members as a place where people of like minds may congregate and strategize. This is not to say that the issue being discussed was not political in nature, but that the Daily Kos community is considered by its members to be a safe place to explore ideas, rather than a place to hone one's arguments against political foes. The conflict case is internal to the community itself, as much as it takes place between its members. The findings developed during this research will later be used in conjunction with an analysis of the Daily Kos ratings system to determine what types of communication the community values. 


\section{CHAPTER 2 - REVIEW OF THE LITERATURE}

The primary finding of a recent Pew Internet and American Life Study was that "the Internet and email aid users in maintaining their social networks and provide pathways to help when people face big decisions" (Boase, Horrigan, Wellman, and Rainie 2006: 1). A recent study released by the Annenberg School of Communication's Center for the Digital Future found that "More than three-quarters of users who went online for political campaign information sought insight regarding issues and candidates about which they were undecided" (Cole 2005: 1). These studies indicate that the use of computer-mediated communication is increasing in almost every area of American life.

\subsection{Conflict and Interpersonal Communication}

In their book Interpersonal Conflict, Wilmot and Hocker define conflict as "an expressed struggle between at least two interdependent parties who perceive incompatible goals, scarce resources, and interference from others in achieving their goals" (1998: 34). Put simply, conflicts happen when human differences become an issue (Littlejohn and Domenici 2001), and the resolution of a conflict can be either constructive or destructive depending upon the communication and conflict management actions that are used to address these differences.

There are numerous classifications of an individual's response to conflict. (Edelman and Crain 1993) classify four types of conflict personalities: the AttackerDefender who sees the other as an enemy who must be overcome; the Accommodator who goes along to get along; the Avoider who typically denies that 
any conflict exists; and the Stalemater who remains locked into a position and refuses to budge no matter what new information comes to light. Additional models include variants of these approaches to dealing with conflict, framing them as either Cooperation/Competition; Avoidance, Solution Orientation and Control; or a constellation of Competition, Accommodation, Avoidance, Concede, and Collaboration styles, in which Collaboration is often determined as the most creative and beneficial of the styles if there is adequate time for it to occur (Wilmot and Hocker 1998: 111). Any of these modes might be appropriate given the situationfor example, when trying to escape a burning building, there is no time for debate, and the competitive "might makes right" mode is the best one if only one person knows where to find the fire escape. It is important not to remove any strategy from consideration, different problems call for different tools.

Constructive communication skills typically involve a constellation of thoughtful and measured actions on the part of the disputants. Specialized forms of active listening and speaking are the rule, with a focus on awareness of both oneself and the other as much as is possible. The founders of the Public Communications Project have developed a skill set for use in team mediation, and for facilitators who seek to assist large groups of people to build community or determine their direction. The LARC skill set is composed of four steps: "listen well to understand one another, acknowledge one another's perspectives, respond clearly with their own point of view, and commit to a course of action" (Littlejohn and Domenici 2001: 104). In this skill set, acknowledgement is a more sophisticated form of 
paraphrasing, in that it involves multiple actions, including restating or reflecting back the previous speaker's words, and then asking for acknowledgement that one's own understanding is correct before responding to the previous speaker (Littlejohn and Domenici 2001).

Additional strategies of conflict management focus on developing and using the following communication skills: speak your mind and heart, listen well, express strong feelings appropriately, remain rational as long as possible, summarize, and ask questions (check your perceptions), give and take, avoid harmful statements (Wilmot and Hocker 1998: 47). It is worth noting that conflicts often have both intellectual and emotional components, and that the ability to manage conflict communication does not deny the existence of strong feelings. Rather, the discussant has the responsibility to express those feelings in a non-harmful manner.

Verbal aggressiveness, when it occurs, is often a sign that a person's feelings have overrun their intellect and can be either a precursor or a stand in for physical violence (Wilmot and Hocker 1998). Attacks on character, insults, rough teasing, ridicule, and profanity are all forms of verbal aggressiveness (Wilmot and Hocker 1998). Taking a break from the conversation or otherwise "cooling off" is often necessary during an especially heated conflict, or one which is complex or seemingly intractable (Butler and Rothstein 2001).

Conflict dynamics can never be understood by looking only at an individual's behavior in isolation; the interaction between the conflictants is what will determine 
the outcome of the conflict, and the interaction they create is often a covert "third party" in any dispute (Wilmot and Hocker 1998).

\subsection{Computer Mediated Communication versus Face-to-Face}

\section{Communication}

The merits and problems of CMC have been discussed for many years. CMC's detractors (see discussion in (Kollock and Smith 1994; Rafaeli and Sudweeks 1997; Riva 2002)) state that CMC is an ineffective medium, fraught with problems in large part due to the lack of extra communication signals which exist in face-to-face (FtF) communication. The lack of non-verbal conversational cues, it is argued, limits the meanings, connection, and understanding that might occur in $\mathrm{CMC}$ (Dorado, Medina, Munduate, Cisneros, and Euwema 2002; Hebert and Vorauer 2003). As well, misunderstandings do abound. For instance, the lack of voice tone with the message causes messages sent as jokes to be perceived as serious, starting online fights because the initial message was misunderstood. This has necessitated the invention of emoticons or smilies (such as $\odot$ or ;-( ) which provide shorthand for communicating emotional tone or context (see (Serfaty 2002; Shea 1994) for examples). The use of ersat $\mathrm{html}$ tags, such as <snark $>$ to connote barbed sarcasm, or <rant> which indicates the author's awareness of message tone, are used within the Daily Kos and other venues. Despite this supposed improvement to communication, some message boards ban their use as an indicator of communicative incompetence (Benson 1996). 
On the other hand, in certain venues such as online feedback in communal work, the lack of extraneous and non-verbal communication cues keeps the focus of the receiver on the message itself, which results in more content-rich communication (Hebert and Vorauer 2003). This has been tested only in certain venues (Hebert and Vorauer 2003), but could also occur in the discussions that take place in the comments area on a political weblog.

\subsection{Participation and Rules Conduct within Online Discussion Groups}

Hosted discussion groups typically incorporate the means to deal with potential conflicts which occur in their spaces within their rules of participation. Commercially hosted groups such as AOL or Yahoo! require acceptance of a codified "terms of agreement" list of rules before one is allowed to participate in the community - the most important rule being that which allows for the exclusion of those who refuse to comply with the posted rules. Privately hosted discussion groups and bulletin boards typically mandate their users' understanding of netiquette, an unofficial list of desirable and undesirable behaviors originally created for Usenet groups, but often codified in hosted discussion groups (Shea 1994). As with the commercially hosted groups, the rules of participation are easily accessible on the site, if not required to be read as a condition of membership (Powazek 2002; Daily Kos, 2006). As well, the site owner may post a warning which threatens to either humiliate or cut off the user who engages in rude, dishonest, or offensive behavior (Haughey 2002). Thus, personal ownership of the community might mitigate the 
possible conflicts which could occur, but it by no means removes all conflict from the venue.

Rules of conduct can also limit who feels comfortable in the community. Depending on the type of leader the community has, these rules may severely limit the diversity of views which are allowed within the community. Consensus theory shows that allowing more people into the discussion arena increases the creativity of the discussion group as a whole, and frequently results in the creation of more and better solutions to problems (Butler and Rothstein 2001; Zafeiriou 2003). The desire for a diversity of people and views to be represented within a community should be balanced by barrier to entry measures that keep participants feeling safe within the group. This is especially important for groups which will also meet face-to-face, such as Minnesota's e-Democracy project, which has been effective in increasing its members' civic involvement offline (Lyons 2002). That group has similar barrier to entry conditions as those found in early Usenet communities, and its archives are not publicly available without registration, which provides a measure of privacy for group members.

\subsection{Self-expression and Conflict Online}

As stated earlier, the fact that $\mathrm{CMC}$ is a depersonalized medium has both positive and negative effects. The depersonalization gives people who might not otherwise connect with others a venue in which their social standing is equal to people with whom they cannot compete offline. However, the online world's absence of physical and social cues can also enable people to feel more comfortable 
to express themselves in ways that they would never do in face-to-face conversation (Kiesler 1997; Wallace 1999), a phenomenon known as the disinhibition effect (Suler 2004).

Flaming and trolling are two instances wherein a user undertakes an abusive conversational tack relatively free of any offline repercussions. Given that the majority of flamers or trollers have the goal of disrupting community interaction, flaming and trolling should not be seen as evidence of a diversity of viewpoints in the community. As with much human interaction, the most important mediator of online behavior is the purpose or intention of the person who goes there (Wallace 1999).

Nothing can be known online about a user unless they make it known through self-disclosure. The research of (McKenna, Green, and Gleason 2002) on the location of one's Real Self indicated that for those online users who might not connect with others offline due to the 'gating effects' of unattractiveness, evidence of social anxiety or physical impediments, the relationships they form online are just as important, if not more so, as their offline relationships. Although stories of falseidentity exist (Turkel 1995), in most online venues, people are who they say they are. Even if they decide to assume a separate online persona, they will still bear the emotional brunt of behaviors and text that is directed toward them. Additional study showed that users who are ignored or snubbed online feel the same way as users snubbed in real life (McKenna and Bargh 1999). Thus, people will be most likely to behave in ways that do not threaten their offline sense of self. 


\subsection{The Effect of Moderation on Communication}

Research measuring the effect of comment moderation on communication has focused on Slashdot, an online community which allows users to offer feedback on each other's commentary. Slashdot's model is unique, in that a limited number of stories are posted each day, and only a small segment of the user population has moderation privileges, which expire in five days' time (Powazek 2002). The focus of those studies has been to evaluate the timing and alleged "fairness" of comment ratings (Lampe and Resnick 2004), whether comment ratings were successful in promoting high- versus low-quality comments to the community view (Lampe and Resnick 2004), and whether the ratings system helps new users acclimatize to the site and improves their participation (Lampe and Johnson 2005). Each of these studies found that the moderation of comments was of benefit to the Slashdot community.

\subsection{Conflict within Online Communities}

The most enduring concern about online communication is that it is needlessly combative due to the depersonalization that occurs when one is a nameless, faceless actor in an unmoderated space (Bellini and Vargas 2003; Davis 1999; Reid 1999; Riva 2002). Indeed, the earliest discussions of online conflict researched the conflicts that took place in Usenet, a collection of unmoderated discussion groups. Its free-for-all atmosphere existed in large part because no one owned, or can own, any Usenet space. The only options people participating in 
unmoderated discussion groups had were either to opt out, or to ask an offender to leave, but with no power to enforce that request.

In spite of this issue, researchers of Usenet have also found ample evidence that online groups perform as flourishing gift cultures, noting that users offered each other free technical support, travel recommendations, medical advice, and other information useful to the community at large (Kollock 1999; Preece 2001; Rheingold 2000). The dreaded "tragedy of the commons" outcome of finite common resources being overrun by selfish individuals is much less of an issue in the Internet space than in a face-to-face venue. When they do participate, community members are more likely to do so favorably rather than unfavorably (Rafaeli and Sudweeks 1997), but as might be assumed, the study of the inevitable conflicts was what tended to inspire the most research.

Unfortunately, a cottage industry of trolling in Usenet meant that not only did some conversations degenerate into flame wars, but that some people actively sought out newsgroups to infiltrate and destroy, via sophisticated strategies sometimes coordinated with multiple users (Herring, Job-Sluder, Scheckler, and Barab 2002). A notable incident consists of a feminist group being infiltrated by a troll (Herring, JobSluder, Scheckler, and Barab 2002). The community was divided in its attempts to deal with the troll, who nearly attained his likely objective of causing the group to disband. While study of this phenomenon has lessened because usage of Usenet has decreased, it is useful to note that the early detractors of Usenet conversations as conflict-rich had many examples of this type with which to prove their point. 
Technology developments within email lists now allow for the presence of a moderator, whose main function is to prevent the list from being inundated with commercial emails often not of interest to the users (commonly known as "spam"), as opposed to mediating conflicts between list members. In blogging communities, moderation may also be built in, as when the site owner deletes comments $\mathrm{s} / \mathrm{he}$ deems add nothing to the discussion, or, as in the case of the study community, when many community members are given the ability to hide comments from the general view. As well, comment ratings schemes (such as the one used in Slashdot, which was the precursor to the one used in Daily Kos) give all community members the ability to rate comments according to their value to the discussion. It is likely that this moderation sets a higher standard for the comments that remain, and reduces the presence of conflict for conflict's sake.

Conflict, however, can provide cohesion in some online communities, as noted by Viviane Serfaty, who states that "in all newsgroups, conflict guarantees the continued existence of the group and its cohesiveness" (2002: 195). Serfaty (2002) described three ways that "benign conflict" within communities results in increased community cohesion: discussion about appropriate norms and behavior within the community, the use of civility and social dominance behaviors (such as sarcasm, irony, and wordplay) to minimize conflict, and the use of visibility strategies to increase a user's presence and position within the community.

The research of Hobman, Bordia, Irmer, and Chang (2002) examines the difference in types of conflict in computer mediated communication versus face-to- 
face groups, but does not expressly look at the way that face-to-face conflict management strategies make the transition to online use, or their impact on communication or conflict within existing groups. It is this gap which this research seeks to fill. 


\section{CHAPTER 3 - METHODOLOGY}

\subsection{Position of the Researcher}

The researcher has been an avid reader of online content for about 10 years, back before the World Wide Web was in the "what you see is what you get" (WYSIWYG) format that it is now, and has viewed the evolution of online communities with both optimism and despair. The researcher is impressed with the ability of $\mathrm{CMC}$ to facilitate asynchronous connections across distance, but was dismayed if not offended by early AOL chat rooms populated by people determined to see how verbally aggressive or scatological they could be. The development of non-commercial personal websites led quickly to blog software which facilitated a new generation of non-technical web-publishers, of which the researcher is one.

The researcher discovered Daily Kos through another now almost equally well-known blog, Eschaton, which was written by the then anonymous blogger Atrios (since revealed to be Duncan Black, a former economics professor who is now a full time blogger). Although Daily Kos was not initially that different from other comments-enabled political blogs, it was not long before the researcher came to believe that the comments community that had developed on the site was worthy of further study.

Because of this belief, the researcher stopped participating in comments threads in roughly October 2003 , choosing to remove herself so that she would not pollute any future observations she might make by participating in the community. At the time of the Pie Fight, the researcher was visiting the site on a weekly basis, 
which provided a moderate level of familiarity with the community and participants, similar to that which could be expected of a majority of readers of the site.

\subsection{Research Focus}

As was seen in the previous chapter, there has been a great deal of research covering conflict resulting from various aspects of the computer mediation of communication, from the examination of behavior in Usenet to empirical studies of face-to-face conflict versus conflict in online contexts. The present research differs from previous research in that it examines the usage of online conflict management strategies "in the wild," investigating the conflict communication and interaction between the members of an established online community resulting from the actions of the site owner.

\subsection{Data Set}

\subsubsection{Site Selection}

The Daily Kos site was chosen for this research because it is currently the most highly trafficked political blog, with about 12 million visits per month, as calculated by Sitemeter. There are almost 80,000 registered members who have posting privileges on the site, but there is no way of determining how many of these members are active readers of the site. More importantly to this research, there is a numerous and prolific comments community that writes comments and journals on the site.

During the time of the Pie Fight, between 5,000-6,000 community members posted at least one comment per week and around 200 individual diaries were posted 
per day (jotter 2005). The site's popularity goes beyond the Internet: both Moulitsas and Daily Kos are frequently discussed in mainstream media outlets such as the New York Times, Newsweek, and Time Magazine. The Daily Kos community is more than merely a gathering of disconnected individuals. Many members have been posting to the community for years, and are highly invested in the perpetuation of good relationships between members as well as good communication on the site.

\subsubsection{Description of Pie Fight Incident}

On Friday June 3, 2005, an advertisement for Turner Broadcasting Network's reality show The Real Gilligan's Island first appeared on the Daily Kos website. This advertisement contained a picture of two women depicting Ginger and Mary Ann from the original television show Gilligan's Island in the middle of a food fight featuring coconut cream pie. Clicking on the image, a pig-tailed Mary Ann licking her finger while gazing seductively at cream-pie-covered Ginger, took the user to a web page containing a video commercial of the show. The commercial featured two women, Mary Ann dressed in short shorts and a low-cut, midriff-baring tie-top, Ginger wearing a low cut gown with a thigh-high slit, each getting progressively more disheveled and aggressive as they threw pies at each other, eventually culminating in their wrestling each other to the ground.

At least one community member posted a derogatory diary article about the advertisement being sexist and thus inappropriate for the Daily Kos site on Saturday June 4. The following day, Sunday June 5, Moulitsas himself posted a diary which took issue with the initial anti-"Pie Fight" diary, and with similar additional 
comments he had received via personal email. Moulitsas ended his post with the admonition that if people did not like it they "could go to other sites (which could certainly use the traffic)," but that he was going to focus on "the important shit". This front-page article unleashed the firestorm of commentary; the comments discussions resulting from it and the subsequent diaries about the Pie Fight (as it had come to be called) are the focus of this research.

On Monday June 6, an even more risqué 60-second "director's cut" of the advertisement was placed on the site. This version was explicitly targeted at mature audiences, and was only viewable between $10 \mathrm{pm}$ and $5 \mathrm{am}$ EST. The new ad, combined with increased commentary about Markos' response to others' criticisms, kept the Pie Fight and discussion going for a week. The final comment in the research corpus was posted on June 13 , nine days after the initial diary.

\subsubsection{Why this Case?}

While this was not the first controversy that the Daily Kos community had experienced, it generated commentary on numerous other well-traveled blogs and even garnered mention in mainstream media (dKosopedia 2006). In addition to highlighting the controversy, the external discussion also demonstrated how wide a read the Daily Kos site enjoys and that many more people know about and reference the community. It also demonstrated that what happens on the Daily Kos site does reverberate past the community, and indeed, of that community's blogroll ${ }^{9}$. It is still

\footnotetext{
${ }^{9} \mathrm{~A}$ blogroll is a list of links to other weblogs which might be affiliated with the blog either through personal connections or topic content.
} 
being referenced within the community itself almost eighteen months after the fact, mostly in the form of jokes seeming to indicate concern about expressing an unpopular view, e.g. "I don't want to start another Pie Fight, but..."

The Pie Fight, comprising as it did issues of sex, community ownership, feminism, and inclusion, not to mention holding a prime position in communal memory, was a ripe locale to examine community interaction about topics that contain a strong emotional component, and thus offered ample opportunity to examine conflict communication.

\subsubsection{Total Number of Authors, Comments, Ratings}

The number of active authors during the week in question was approximately 5,000 , and 1,820 community members (about $36 \%$ of the active user base) participated in some fashion. Given these numbers, a conservative estimate of the number of lurkers who read the Pie Fight diaries would be 3,180 members. However, because the site experiences approximately half a million unique visits a day, it is impossible to know how many people - community members or not - viewed the diaries in this study.

The Pie Fight discussions took place in 28 diaries, containing 7,238 total comments in 1,355 total comment threads, written by 1,279 total authors ( $70 \%$ of the total participants, with $44 \%$ of those participants contributing 3 or more comments). 18,568 comment ratings were made by 1,294 community members $(71 \%$ of the total participants, with $56 \%$ of those participants rating 3 or more comments). 753 of the participants $(41 \%)$ made both comments and ratings, 526 participants (29\%) wrote 
comments but did not rate any comments, and 541 participants (30\%) rated comments but did not write any.

The diaries which were chosen for this case study contain a minimum distribution of comment ratings from 1.00 to 4.00 , and have not already been selected for use in other research. This ratio is important because comments which receive ratings have received more attention by definition than comments which have not received a rating. That a user has taken the time to rate a comment, either positively or negatively, means that the comment was seen as deserving either special censure or special praise, outside the normally expected value of comments on the site. It is important to note that although there was a distribution of ratings, not all of the ratings were visible or considered in this research.

\subsubsection{Definition of Terms}

The terms below are more fully explained in Appendix B, but are included here as a basic introduction.

\section{Diary}

A diary is a story designed to inspire discussion. Diaries are typically much longer than comments. In the Daily Kos community, each member can only post one diary per day, so it is understood that authors put more care and attention to creating a diary than they might to writing a comment. 


\section{Comment}

A comment is a written response to the diary, or another comment, posted on the site. Aside from time constraints, there is no limit to how many comments a Daily Kos member may post in a day.

\section{Comments Thread}

A thread is a collection of related comments. Comments threads are typically arranged in chronological order, and each subsequent comment is slightly indented to the right and below the comment to which it is responding. Each comment that responds to the original diary begins a new thread. More than one comment can be posted in response to a parent comment located earlier in the thread, but each comment can only be directly threaded to a single earlier comment to which it is responding (See Figure 2).

\section{Rating}

Each member of Daily Kos may rate a comment using the following criteria, which are posted in a dropdown menu just below the author's name, and to the right of the "Reply to This" link: 1 - Unproductive, 2-Marginal, 3 - Good, 4 - Excellent (See Figure 2). When a member gives a rating of 1 or 2 , they are said to be "downrating" a comment. A convention of the site is that two people must rate a comment for the rating average to be visible to the community on the main comments page. In cases where only one member rated a comment, the display changes from (none/0) to (none/1). Interested readers can click on the (none/1) link 
to see what the rating was, but the rating is not displayed to the casual reader. This information was not analyzed within this research.

\subsection{Research Sample Selection}

The researcher chose to examine conversations that occurred within two online diaries discussing the Pie Fight conflict. Because this research is a pilot for future dissertation research, the diaries chosen for use in that research could not be considered for use in the present research. The two diaries were chosen because they represent different viewpoints on the conflict, although both diarists were concerned about the effects of the Pie Fight on the community. Both diaries were written at about the same time, early to midway through the conflict, and are diary number 10 and number 11 of the 28 total diaries. These diaries will not be included in subsequent research, and had not been read prior to the current research.

Each diary contains at least some ratings other than 4.00 , although the actual displayed ratings were rarely other than 4.00 , meaning that those who downrated were in the minority. Both diaries were written by men, one of whom was well known in the community at the time. The researcher's criteria of a variety of ratings and a minimum of 100 comments per diary, as well as the selection of diaries to be used in subsequent research, severely limited the choice of appropriate diary.

\subsubsection{Comments Threads and Conversation Threads}

A comments thread is a string of messages that are arranged to appear as a conversation as the reader reads down the page. For the purposes of analysis, the researcher created the following organizational scheme, comprising measures of 
Comment Number, Parent Comment, Thread Number, and Depth within the comments thread. The originating diary is treated as Comment 0 , Depth 0 , and has no Parent. Each comment that responds to the original diary has a Parent of 0 (the original comment number), begins a new thread, and is at Depth 1 . Thus, the first message in a diary would be labeled as Parent: 0 , Depth: 1 , Comment: 1 , and is the beginning of Thread 1 . The responding subsequent message would be Parent: 1, Thread: 1, Depth: 2, Comment: 2, and so on down the line. The next comment having a Parent of 0 would begin Thread 2, and have Depth: 1 , and a Comment number reflecting its location within the dataset.

All comments within the data set are date and time stamped, but are numbered in the order in which they appear on the page. In some cases, a comment that was posted later in the conversation will appear earlier in the data set.

A conversation thread is a collection of comments having a Depth of 1 or higher which are located within the same thread, and which contain the participation of at least two people, who speak at least two times within the conversation thread.

\subsubsection{Unit of Measure}

For this study, the initial unit of measurement was the individual comment message. This measure has several advantages. As stated by Rourke, et al (2001), standardizing on the message creates an objectively identifiable unit, whose parameters have been created by its author. Even though there may be multiple meanings embedded within each message, there is no question about the centrality of the message as the unit of analysis. 


\subsubsection{Limitations - Hidden Comments}

Although one limitation (noted above) is that two ratings must occur to publish a rating, the other is that comments with an average rating of less than 1 (e.g. any comment which receives a 0 and a 1 , or multiple 0 's in combination with any sum for which the average will be less than 1) is hidden from view of the average user. In prior readings of the site, the researcher discovered references to these hidden comments that testify to their volubility and rancor—several members stated that that they "need[ed] to shower" after reading them. Study of these hidden comments would be a very useful adjunct to this research, showing as they would which comments were deemed unproductive by the community's Trusted Users. However, they are not publicly available, and several communications to the site owner requesting access went unanswered.

\subsection{Data Analysis Procedure}

The researcher used a generic qualitative approach, described in Creswell (2003: 190-195) to do the analysis within this case study. Following the collection and delineation of the dataset, the researcher read through the dataset multiple times to gain a general sense of the data. The researcher then began a more detailed analysis, which was followed by a description and generation of themes which were analyzed and then interpreted.

\subsubsection{Selecting Conversations}

To find evidence of conversation within the diaries, the researcher first read through all comments in each diary, making note of which comments threads had the 
participation of at least two different people who spoke at least two separate times within the thread. Out of 38 total conversation threads in Diary 10 , and 17 total conversation threads in Diary 11 , a total of twelve conversations were found, six in each diary. These conversations are listed in Appendix A, which also includes the URL of a web page which links to each diary in its original context, the diary text itself, and the twelve conversations that were selected for use in this research.

Although both diaries contained ample discussion and conflict, the researcher determined that, for a real conflict to be presented and resolved, at least one dialogue event involving back-and-forth conversation between at minimum two people was required. The requirement of the presence of the same person at least twice within the specific conversation thread further limited the threads that were to be analyzed within this research.

It is important to note the existence of multiple subthreads within individual conversation threads, meaning that there are more than twelve individual conversations that took place. An additional finding of this research is that participation within these subthreads resembles the way individuals move through different conversations at the same party. An individual might appear two times within a single conversation, but only one time in each subthread. Initially, these subthreads were going to be considered as separate conversations, but subsequent coding required these subthreads to be considered as occurring under the umbrella of the main thread conversation. 


\subsubsection{Thematic Content Coding}

After the conversation threads were selected, the research coding procedure involved reading each conversation thread through several times. The initial readings enabled the researcher to get the gist of each comment in relation to its context. Subsequent readings were used to determine content, tone, or purpose of the comment, and each comment was then coded individually in consultation with similar content analysis conversation codes such as those found in Bales' (1950) small group interaction research, which focused on purpose and tone of the utterance.

Themes were determined based on an emergent system after several reads through, after comments were coded. Subsequent reads through the threads were undertaken to get a feel for the progression and tone of the conversation, and to discover and delineate different patterns of interaction. 


\section{CHAPTER 4 - ANALYSIS AND DISCUSSION}

\subsection{Findings on Communication Style}

\subsubsection{Finding 1 - Quoting, not Paraphrasing}

In all communication there are two conversations - the one that occurs and the one that is understood. In offline communication, the meta conversation takes place within paraphrasing, the purpose of which is to check one's assumptions of the other's intentions or meaning before proceeding with a new statement or communication.

Paraphrasing is a multi-step process, in which the speaker and listener have active roles. After the speaker has finished articulating their thought, the listener is responsible for rephrasing the speaker's words, conveying the listener's understanding of the speaker's meaning, receiving acknowledgement from the speaker that this understanding is correct, and then articulating a response to this information. The listener and speaker roles are then reversed, and the cycle begins again. More than merely a parroting of each other's words, paraphrasing allows for a more complete understanding and depth of communication between the speaker and listener.

Within the Pie Fight, the cut-and-paste "paraphrasing" that occurred consisted of directly quoting a previous discussant's words back at them, without the meta-communication or interpretation that takes place in paraphrasing in face-to-face contexts. The result was is that previous commenter's words are interpreted and responded to in one step. The break or thoughtful pause that is involved in 
paraphrasing did not exist in this process. Paradoxically, the time needed to respond to a previous commenter appears to have been used to stoke the fires of disagreement, as the previous comment was responded to by a person who had already determined what that person meant, and responded accordingly. This occurred in the following ways:

The original statement is quoted, but converted to a question by adding a question mark:

Kos got greedy? Diary 11, Comment 12.

Exactly how does "Call a waaaambulance" even begin to "realize people have different opinions than you and its OK. " Diary 11, Comment 20.

"Slap Me? Why?" Diary 11, Comment 22.

In each the above cases, the commenter clarified their interpretation in the subsequent sentences, but the initial statements were issued as challenges to the original commenter.

In one case, quoting the previous commenter's words out of context was used as evidence enough that their intent was suspect:

And when you say 'frigging, ' what the hell does that mean? Huh?

Huh? Diary 11, Comment 10. 
Block quotes were used for more targeted challenges or disagreements, as seen here:

"It is fine if you disagree with me and my views, but I am willing to hear yours, you only want to marginalize or shut mine up. " [Diary 11 , Comment 64, shirlstars]

If you think the latter part of this is true, it is, I would say, prima facie evidence that the former part is not. Diary 11, Comment 68 .

However, the above sentence was extracted out of a lengthy, six-paragraph comment titled "the problem is being marginalized consistently." Perhaps needless to say, the author of Comment 68 did not engage shirlstars in any of her more substantive points.

In one case, which demonstrated the commenter's awareness that she might have been unclear, the commenter preemptively quoted herself, clarifying her intent before it could be misinterpreted:

“ALso, Paradox-don't take that line (foisted upon me) personally.

Frankly, I think this is an important diary, and you have said it well." Diary 11, Comment 44.

In the online world, paraphrasing to communicate understanding is perhaps seen as tedious by people who are inexperienced in its use or purpose. Instead, CMC enables conversants to directly quote each other by means of cutting and pasting from previous comments. Rather than re-typing what the previous poster has said, 
the discussant can simply cut and paste the statement(s) to which s/he wishes to respond. This quoting convention likely results from earlier quoting conventions in online communication which were designed to save bandwidth (Shea 1994), or to indicate to whom or to what comment someone was responding within a nonthreaded discussion space.

Although the Pie Fight took place within a threaded discussion space, the use of direct quoting rather than paraphrasing was the norm. Commenters seemed to use these direct quotes to prove the original writer wrong, even when this ostensibly occurred in the context of asking questions about the original statement. What appeared to be paraphrasing had the opposite effect of escalating the conflict rather than promoting understanding between conversants.

Unfortunately, this cut-and-paste response process often led to an increase in miscommunication, and the person who was misunderstood rarely returned to correct the record. The metadiscussion that occurred was about "what the conflict is about," not about the way in which people were understanding each other. Rather than use this practice to gain a better understanding of the previous commenter's meaning, people were much more likely to use direct quotes from previous posters to advance their own arguments.

\subsubsection{Finding 2 - Apologizing}

Throughout the sixteen threads, there were three incidences of apologies that took place within conflict threads. Apologies were rare, but were more likely to occur when only two people were involved in the conversation, such as in Diary 11, 
thread 13. This could also be the because of the personality of the conflictants themselves.

The presence or lack of apology within the diaries could have something to do with the cues filtered out theory. Alternately, the use of apology language might not be as appropriate for the entire Pie Fight as for the micro conflicts or misunderstandings that occurred.

There was one case in which a lack of understanding of the medium on the part of other commenters was raised, in Comment 21 in Diary 11:

"We are communicating over the Internet, which is prone to misunderstanding, misreading, and all other forms of general miscommunication. This leads to huge fights, flames, cursing, etc.

But, to be a literate blogger, you need to accept this, and not take it personally."

However, this commenter is speaking about the response to his Comment 2, in Diary 11, which was titled "someone call the waaaambulance", and the lack of forgiveness of the frustration which caused him to make it. Considering his word choice, it is doubtful that the medium is the sole factor to blame for the response that the commenter received. For the most part, community members rarely engaged in medium blaming for the conflict or escalation that had occurred. 


\subsubsection{Finding 3 - Using "I" or "You"}

The research of (Arguello, Butler, Joyce, Kraut, Ling, and Wang 2006) indicated that commenters in online discussion groups who used first person pronouns were more likely to receive a response, and additionally that pronoun use served to create in- and out-group boundaries. This research found no difference in the type of further communications such "I" and "You" comments brought about. Indeed, within the main corpus of Diary 11 and 12 , the majority of comments read "down the line" appeared to be independent of the previous comment, rather than responding directly to it.

However, there was much more of an "I" and "You" sequence of interaction, versus people following up I-statements with additional revelations of their own.

The use of "l" statements is believed to bring about increased understanding if both (or all) conflictants use similar sentence construction to convey their understanding (Edelman and Crain 1994). What happened in the Daily Kos is that an "I" statement was not followed by a second "I" statement, but by a "You" statement that addresses the community at large, but leaves the first speaker somewhat adrift. There did not seem to be any recognition that the initial user's " $\mathrm{I}$ " statement was responded to and understood by the subsequent speaker. Rather the next speaker chose to take an accusatory tone toward the remaining readers of the thread in question. Needless to say, this does not engender a deeper understanding among the readers and discussants within the conversation thread. 


\subsubsection{Finding 4 - Referencing Emotion}

Emotion, when mentioned in the conflict threads, typically was either a confession of personal anger or an admonition that "other people" need to cool off or cool down. The comments following these tended toward amplification: "Yes - I'm angry too" or "The community is more important than this." Rarely was a confession of anger followed by a comment that expressly denied the right of the commenter to feel this way.

In one interesting case, a commenter who suggested that people who were offended needed to "call the waaaambulance" (Diary 11, Comment 2), stated less than 15 minutes later that people need to "be gentle and don't carry grudges. And relax. And breathe. And realize people have different opinions than you and its ok" (Diary 11, Comment 19). When challenged about his earlier waaaambulance comment, the author blamed it first on frustration, and second on the Internet's being prone to misunderstandings and misreading. He admonished his challenger not to "take it personally." This author also agreed with Markos that people who didn't feel that the site was meeting their needs should leave. Thus, his call for calm was really a call for people to put up or leave. It is often very easy for people to recommend calm when they are in the dominant position or are not otherwise feeling attacked or emotional.

\subsubsection{Finding 5 - Switching Personalities}

Within the Daily Kos, conversation is multifaceted and constantly evolving. While some people may "pile on" and join condemnation of a person or comment, 
others seemed to undergo changes of opinion regarding a single issue within the same diary. However, this transformation was demonstrated only in subsequent comments within the conversation. Only rarely were these changes of heart incorporated into the original thread - users did not often go back to the same thread to explain their new thinking - rather, their subsequent comments displayed an evolution of behavior or feelings.

Interestingly enough, the asynchronous timeframe allowed people to take multiple points of view. At Comment 117 in Diary 10, Earl was definitely angry at Delaware Dem, yet at Comment 111 (which occurred about twenty minutes later), Earl "completely agrees" with Delaware Dem. His viewpoint evolved as demonstrated by comments 102 and 136 (note that comments that appear numerically earlier in the thread were actually written later, chronologically).

It's possible that people are more likely to change when they're involved in a distributed conversation than when they're involved in a face-to-face interaction. This is likely because they are not in public and so do not feel the need to maintain a face in the same way that face-to-face interaction happens. As well, they might be able to take in multiple points of view more rapidly because, although 10 people may be talking "over" each other, the presentation of their statements can be incorporated in the reader's own time. Rather than being pressed to respond immediately, the reader/participant of a distributed conversation can adapt to new information and formulate a more considered response. 
This multiplicity played out in Diary 10 , comments thread number 31 , wherein a poster, DHinMI, made a sexist, but joking, response to a woman who was trying to explain the context of the Pie Fight ("Ahhh, you just got hysterical. You're probably a little too emotional. <ducks to avoid being hit>" Comment 119) which was ill-received by Cathy, the woman to whom he had responded, and by another woman who entered the conversation specifically to upbraid him for his use of sexist language (SallyCat). In the first instance, Cathy returned a few minutes after DHinMI explained he was making a joke, to admit that she had overreacted, and all was forgiven by DHinMI. In the second instance, which occurred earlier chronologically, the conflict escalated rapidly - DHinMI argued that SallyCat just "want[ed] to pick a fight" and stated in two subsequent comments that she should just leave the community altogether, although she had stated earlier in the diary these would be her "parting" comments. This rapid escalation could be because of behavior that was observed within other diaries or threads, because of DHinMI's previous experience with SallyCat, or for some other reason that the researcher cannot know. In the same way that the diffusion of responsibility allows people to behave in ways they might not choose to if they feel as though their actions are on stage for the world to see, distributed conversations allow participants to be different with each encounter. The researcher or attentive reader is able to see this because of the historical record, but there is little overt recognition of this behavior within the conversations by the conversants themselves. 


\subsubsection{Finding 6 - Extended Leave-taking}

Interestingly, SallyCat, Maryscott, and others who talked about the fact that they had left the community, were still posting comments within a Daily Kos community diary. While there is nothing to stop a member from simply disappearing from the community altogether, at least some in the Daily Kos chose a different strategy for departure, a long goodbye rather than an abrupt disappearance. This extended leave-taking allows members to express a wish to stay connected with various community members, or post a "forwarding address" to a new individual or community site. Because many members in the Daily Kos post under pseudonyms and don't need to include contact information, the community members must be very intentional about securing those connections they would like to keep before they leave, which might require several goodbyes in several different places.

\subsection{Findings on Conflict Minimization or Avoidance}

\subsubsection{Finding 7 - Minimizing Conflict}

The use of civility and social dominance behaviors such as sarcasm, irony and wordplay to minimize conflict or bring online communities together (Serfaty 2002) is seen in certain aspects of the conversations that occurred in the data sample. While the use of profanity or sarcasm might in other contexts be termed verbal aggressiveness, in certain cases this communication strategy seemed to result in additional humorous remarks or other indicators of appreciation or agreement. When this behavior did occur, it seemed to occur almost exclusively on the part of people 
who were part of the "in" group, which seemed also to be the group that seemed to believe that the Pie Fight was overblown or otherwise unimportant.

Evidence of wordplay or irony occurred in numerous instances throughout the dataset, often occurring when a serious or explanatory comment was followed by a joke, sarcasm, or simply an unrelated remark. There was one instance in which a wordplay strategy backfired spectacularly. Although it did not occur in this dataset, it was discussed as an important factor in people's feelings and behavior. Mr. HinkyDink coined the term Menstruating She Devils to describe the women who took offense at Markos' diaries. This offensive appellation was said to be the impetus for the creation of a new blog of the same title, which was created and maintained by women who chose to leave the community. Mr. HinkyDink referenced his apology for this unfortunate and misunderstood joke in Diary 11 Comment 47, and explained his thought process and chagrin more thoroughly in Comment 49. His apology was well-received and appreciated. In a lighter instance of wordplay, Maryscott coined the neologism Kostroversy, giving her the last word within a somewhat heated discussion in Diary 11, Thread 1.2.

On a number of occasions a subsequent dismissive comment seemed to undercut or circumvent the original author's obviously concerned communication. It is quite likely that the respondents were seeking to lighten an atmosphere that had seen a great deal of contention in the previous days, but this strategy also prevented further or more in-depth discussion of the original author's communication. The explanatory comments which were dismissed or derailed had very high numbers of 
high ratings (see Diary 10, Comments 92, and 118, and Diary 11 , Comments 36 and 42), so the authors of the comments knew that they were appreciated. However, it is possible that they, and the community members who held the same viewpoints (and who may have indicated this by uprating these comments within the thread rather than composing their own responses) felt dismissed and unwelcome in the community because of the lack of substantive follow-up to their carefully written or heartfelt posts. A future analysis of the subsequent off topic comments might show which areas or viewpoints cause the most confusion/desire to change the subject.

\subsubsection{Finding 8- Deleting Content}

Within Diary 10, Comment 92, Armando, a front page diarist and esteemed community member, called upon the Delaware Dem to delete the diary and all its comments, almost fourteen hours after it was posted. Delaware Dem's response indicated that he recognized that he was being singled out for rare attention from a diarist who has written equally, if not more inflammatory, statements. Since the diary still exists we know that the diarist did not follow this advice, and this research is richer for it.

The deletion of diaries which were presumably seen by their authors as unproductive or offensive, along with all of the comments within them, occurred two times within the Pie Fight, although the diary titles and author information still exist in the dKosopedia. As well, the comments that were attached to the site owner's two diaries on the subject are no longer visible from the main site (the researcher has preserved these original comments for study in future research). Although it is 
tempting to believe that this "disappearance" of comments might have calmed the situation somewhat, it is equally possible that the members who felt slighted or unheard by the community felt further marginalized by the decision to eradicate any evidence of their dissent.

The question becomes: is it better to have conflict conversation to refer back to, or does its presence merely "fan the flames," as Mike S stated in Diary 10, Comment 104 . One of the benefits of CMC is the facility with which a communal memory can be maintained. While this memory has important benefits, the fact that several diaries and their attached comments were deleted speaks to a desire for the community to return to "the way things were before," as if such a memory wipe is possible. This disappearance of record, whether undertaken for reasons of vanity or a desire to calm, has troubling implications for conflict resolution within online communities in general.

This ability to easily ban a person (or a site) from your experience is a unique feature of online life. One can essentially refuse to be exposed to any person or idea that they don't want to see, especially if the only connection with them was via a comments board. In the Daily Kos, this ability to delete intractable conflicts or a stream of arguments with which one disagrees, rather than work to resolve or understand them, is power which should be undertaken only in the most extreme circumstances. A better solution might be to suspend access to such diaries for a period of time, to allow members the opportunity to cool off, rather than removing all evidence of the altercation. 


\subsubsection{Finding 9 - Threatening to Depart, Taking a Breather}

Maryscott O'Connor, also known as MSOC within the dataset, stated on numerous occasions in Diary 11 that she was or would be "taking a breather" from the community in the wake of negative ratings and other criticisms she received as a result of her discussions about the Pie Fight. Although an analysis of whether or not she really took a break from the community is beyond the purview of this study, her decision to mention that she was taking a break from the site, while continuing to participate in the conversation in Diary 11, was an interesting one to observe.

In one other case, SallyCat mentioned that she would be leaving, but referenced a "parting agreement" with Armando in Diary 10, and made four subsequent comments in the next 45 minutes. It is doubtful that SallyCat is the only person who chose this type of action; indeed, other references to real or imagined GBCW ("Goodbye Cruel World") diaries indicate that this extended but intentional leave-taking is a common practice on the site. When coupled with other references or discussions of diarists being asked to leave or to delete their diaries, it's not surprising to discover that people wanted to include either parting shots (SallyCat) or explanations for their impending absences, especially considering their visibility within the community (Maryscott).

This is similar to the "cooling off" period that is recommended in interpersonal conflict resolution \& consensus discussion. It is also possible, giving Maryscott's usual posting level on the site, that her 17 comments within the research dataset did represent a reduction from her usual number of comments. 


\subsubsection{Finding 10 - Departing Permanently}

Surprisingly, there were a number of comments indicating that the disagreement was so damaging that participants were taking their leave of the community. The underlying hopelessness behind this gesture was directly remarked upon by diarist paradox, whose "Is Plutonium Page gone too?" (Diary 11) diary sparked a great deal of impassioned discussion, both about why various community members (predominantly women) left, and about the reasons other people chose to remain, in spite of their disagreement with the site owner. The extremes in tone within conversations about peoples' immanent departures indicate that this exodus was very important to the community, with several commenters noting that the departure of a segment of the community boded ill, that the site would be the poorer for it, and that the community (or Markos) should've taken steps earlier to prevent it. Other commenters, who seemed to agree with Markos, did not seem to feel beholden to community members, nor to making the space an inclusive one. These responses tended toward reminding people that the Internet has room for everyone, and that any member was free to create their own website if they felt unwelcome at the Daily Kos. This is obviously true, but it ignores the fact that the Daily Kos community is a unique creation that is not easily replicated. Sadness at distancing oneself from the community was an important (though not always deciding) factor in people's decision to stay or leave the site. 


\subsubsection{Finding 11 - "Invitation" to Exit}

The self-described exit comments are not the same as comments which were in effect asking or demanding that the offender leave. This is different from the strategy of dealing with trolling, because communities dealing with trolls are often told to "ignore" them. Calls for community members to leave the Daily Kos were surprisingly frequent, even when they did not reference any specific member directly. This could be because there is even more of a community face, so this preemptive ostracizing could have more weight behind it. It could be because in this case the "trolls" were already established as contributing members of the community, whose comments couldn't easily be hidden by getting downrated. The disputants in this conflict didn't appear out of nowhere, so they had to be asked to leave rather than merely ignored. This also served as more of an indicator that "their kind" was no longer welcome in the community. This likely has something to do with the power or weight of ostracism to a member of a vibrant community versus the relative disempowerment of ignoring a troll who is not a community member, and hoping that they will go away.

\subsection{Findings on Ratings Behavior}

\subsubsection{Finding 12 - Ratings to Support}

The comments that received ratings of 4.0 were predominantly explanatory meta-communication about the conflict itself, and oftentimes describing the writer's sadness or anger about the situation, specifically about the loss of very good people who were integral to the site. That these comments were often followed by dismissal 
or jokes, as noted above, might have been mitigated by the high number of ratings they did receive.

The use of ratings in this case could be a means for the minority group to weigh in on the discussion without taking the risk of expressing things themselves, or alternately, adding more fuel to the fire. Unfortunately, the dataset did not include identifying information of the raters, so there is no way of knowing whether people who posted ratings also wrote comments in this discussion. However, the pattern of ratings and the viewpoints that they appear to support or compliment are very much on the side of the people who were against Markos' defense of the ad, sad about the perceived negative changes to the site, or expressing dismay about the discourse of the conflict.

\subsubsection{Finding 13 - Ratings to Threaten}

Ratings were used and perceived as punitive in some cases - even the mere mention of giving someone a zero rating brought commenters who were not directly addressed into the discussion. In Diary 10, SallyCat made three mentions of troll rating: In Comment 94, she stated "In keeping with the spirit of understanding I haven't troll rated..." In Comment 125 she mentioned that her "finger [was] twitching on ' 0 ", and in Comment 128 she stated that the zero was "aimed, but not fired." The responding comments were, "Get off the zero" Diary 10, Comment 126, and Comment 133 in the same diary, which stated "Go fight somewhere else, and quit abusing the ratings system." Interestingly, SallyCat never actually gave anyone within the research diary a zero rating, though she stated that she had given three or 
four during the course of the Pie Fight. Although SallyCat was a Trusted User, her mention of potentially using this power to moderate the tone of the discussion brought out an accusation of abuse. Zero ratings were clearly treated very seriously and respectfully by the discussants, which seems to be at odds with the demand that SallyCat, who confessed to considering to zero rate but did not do so, should leave the community for ratings abuse.

\subsubsection{Finding 14 - Ratings to Punish}

In four cases, an average comment rating of less than 4 occurred. In Comment 1 , Diary 11 , a writer used sarcasm to express his sadness about the people who left, by negatively commenting on the "frat boy Dems" who would "fill the void' left by the departing women. This comment got 17 ratings, the most of any comment in the diary, but the average rating was 3.00. Only one person, ogre, explained down-thread why he gave an "unproductive rating"[of 1]: he felt that sarcasm is easily misunderstood, and that the environment was already too charged up to support such language (Comment 8, Diary 11).

The comment immediately following (Comment 2, Diary 11) received an even more punitive average rating of 2.33 given by 9 people, likely for its use of name calling and offensive sarcasm to deride the people who left as needing to "call the waaaambulance." The third comment was titled "Be a man" and included a lengthy, informative treatment of the "Dads and Daughters" advocacy group, but also called Delaware Dem, the author of the diary in which she was posting, a "Loser." This comment received an average rating of 3.33 , by 3 members, which 
means that mathematically it got either two 4's and a 2, or two 3's and a 4. The fourth comment, by GussieFN in Diary 11, received a 3.5 rating from two members, which probably was the result of a response that could have appeared unnecessarily combative, escalating, or challenging to Maryscott and her use of the word "frigging."

These instances seem to indicate that the punitive ratings that occurred here truly were used as a statement on unproductive language, rather than a difference of opinion on the part of the raters. An actual analysis of the distribution of the ratings would give a more precise understanding of the community's feelings about these comments, but was not available within this dataset.

\subsection{Conclusion}

The examination of conversations, in addition to looking at each comment in isolation, enabled the researcher to find patterns of interaction that help to shed a light on the conflict communication that took place within the community during the course of the two diaries. Although this research did not seek to discover exactly why this conflict was so divisive to the community, the previous findings do yield clues about the types of communication that might have made a certain segment of the population feel unwelcome. Even if, as reported on the dKosopedia, membership was at an all time high within the following months, there is no doubt that the Pie Fight has had lasting effects on all who were involved in its discussion. 


\section{CHAPTER 5 - LIMITATIONS AND FUTURE RESEARCH}

\subsection{Limitations of the Research}

The small sample size limits the applicability of these findings without further research in another venue. Additionally, the numerous specialized capabilities of the Daily Kos site, such as the ability to rate comments, the ability to remove comments from view of the community members, and even the new ratings system, will make future comparisons somewhat constrained.

The removal of certain comments from the discussion, although instigated by Trusted Users of the site, hindered the ability of the researcher to examine the full range of conflict conversation. There is no way to determine what types of comments were removed from the discussion, whether they were truly unproductive or merely irritating to a Trusted User who was having a bad day. It is possible, though perhaps not probable, that very provocative and soul-searching questions were seen as too disruptive for the community, rather than as openings to increased information and exploration of the conflict.

Because this research relied on the analysis of pre-existing communication, there is no way of knowing how comments were perceived by the reader, or why certain people didn't return to respond to the people who responded to them, within the conversation threads. A non-response could indicate the writer was angry, hurt, or merely too busy to check back on the conversation. Knowledge of an individual's context would have greatly aided the interpretation and analysis of the observed behavior. 
A limitation of the current research is that it was out of the scope to determine how many comments were written by people who were taking selfproclaimed breaks from the site, or who were leaving outright (though not before weighing in within at least one diary to describe their reasons for leaving). Additional research would also show whether or not the people leaving the community wrote "Goodbye Cruel World" (GBCW) diaries, which have become a convention for site participants who leave the site to post elsewhere or to go offline completely.

\subsection{Future Research}

In addition to the previous chapter's findings, this research yields several interesting questions which are beyond the scope of the study. A longitudinal analysis of participation by those community members whose comments were negatively rated or challenged by subsequent commenters would be of interest to measure the regulatory effects of the ratings scheme. If those members remained active, is there evidence that they "learned" from the ratings their comments received, and are now positively contributing members, or are they still participating in ways that garner only negative attention?

A similar longitudinal study might look at those people whose comments indicated that they were leaving the community, or taking a break, to determine how many remained absent from the community in the following months.

An in-depth interview study of members who were angered or distressed by the Pie Fight or the communication about it could offer insight into what happens to 
members of a minority group within a conflict. Questions such as whether they chose to leave the community, why they chose to do so, and where they ended up could give an indication of coping strategies in the face of divisive or overwhelming conflict. Questions regarding return after departure and the reasons for doing so could increase understanding of what makes people leave communities of conflict and what compels them to return. Finally, interviewing the people who chose to stay in spite of their disagreement with Markos or other community members can offer insight into how people might choose to stay connected to people with whom they know they do not agree, and the strategies which make such connection possible in the face of disagreement about key issues. 


\section{REFERENCES}

Arguello, Jaime, Brian Butler, Elisabeth Joyce, Robert Kraut, Kimberly S. Ling, and Xiaoqing Wang. 2006. "Talk to Me: Foundations for Successful IndividualGroup Interactions in Online Communities." in CHI 2006. Montreal, Quebec, Canada.

Bales, Robert F. 1950. "A Set of Categories for the Analysis of Small Group Interaction." American Sociological Review 15:257-263.

Bellini, Carlo Gabriel Porto and Lilia Maria Vargas. 2003. "Rationale for InternetMediated Communities." Cyberpsychology and Behavior 6:3-14.

Benson, Thomas W. 1996. "Rhetoric, Civility, and Community: Political Debate on Computer Bulletin Boards." Communication Quarterly 44:359-378.

"The Blogging Iceberg - of 4.12 Million Hosted Weblogs, Most Little Seen, Quickly Abandoned" 2003, Retrieved December 11, 2003

(http://www.perseus.com/blogsurvey/thebloggingiceberg.html).

Blood, Rebecca. 2002, "Weblogs: A History and Perspective." Pp. 7-16 in We've Got Blog: How Weblogs Are Changing Our Culture, edited by J. Rodzvilla. Cambridge, MA: Perseus Publishing.

Boase, Jeffrey, John B. Horrigan, Barry Wellman, and Lee Rainie. 2006. "The Strength of Internet Ties: The Internet and Email Aid Users in Maintaining Their Social Networks and Provide Pathways to Help When People Face Big Decisions." Pew Internet and American Life Project, Washington, DC.

Butler, C.T. Lawrence and Amy Rothstein. 2001. On Conflict and Consensus: A Handbook on Formal Consensus Decisionmaking. Takoma Park, MD: Food Not Bombs Publishing.

CmdrTaco. 2003, "Slashdot FAQ - Comments and Moderation", Retrieved November 30, 2006 (http://slashdot.org/faq/com-mod.shtml\#cm700).

Cole, Jeffrey. 2005. "Highlights: Digital Future Project 2005 (Year 5)." Los Angeles, CA: USC Annenberg School Center for the Digital Future.

Creswell, John W. 2003. Research Design: Qualitative, Quantitative, and Mixed Methods Approaches. Thousand Oaks, CA: Sage Publications.

Davis, Richard. 1999. The Web of Politics: The Internet's Impact on the American Political System. New York, NY: Oxford University Press.

dKosopedia. 2006, "Pie Fight", Retrieved November 30, 2006

(http://www.dkosopedia.com/wiki/Pie_fight). 
Dorado, Miguel A., Francisco J. Medina, Lourdes Munduate, Immaculada F. J. Cisneros, and Martin Euwema. 2002. "Computer-Mediated Negotiation of an Escalated Conflict." Small Group Research 33:509-524.

Edelman, Joel and Mary Beth Crain. 1993. The Tao of Negotiation: How You Can Prevent, Resolve and Transcend Conflict in Work and Everyday Life. New York, NY: HarperCollins Publishers.

Grossman, Lev 2006. "The People's Network." Time, November 13, 2006, pp. 61-65.

Haughey, Matt. 2002. "Building an Online Community: Just Add Water." Pp. 201208 in We've Got Blog: How Weblogs Are Changing Our Culture, edited by J. Rodzvilla. Cambridge, MA: Perseus Publishing.

Hebert, Brenda G. and Jacquie D. Vorauer. 2003. "Seeing through the Screen: Is Evaluative Feedback Communicated More Effectively in Face-to-Face or Computer Mediated Exchanges?" Computers in Human Behavior 19:25-38.

Herring, Susan, Kirk Job-Sluder, Rebecca Scheckler, and Sasha Barab. 2002. "Searching for Safety Online: Managing 'Trolling' In a Feminist Forum." The Information Society 18:371-384.

Hobman, Elizabeth V., Prashant Bordia, Bernd Irmer, and Artemis Chang. 2002. "The Expression of Conflict in Computer-Mediated and Face-to-Face Groups." Small Group Research 33:439-465.

Horrigan, John B. 2003. "Consumption of Information Goods and Services in the United States." Pew Internet and American Life Project, Washington, DC.

jotter. 2005, "One Year of Participation at Daily Kos", Retrieved November 30, 2006 (http:/www.dailykos.com/story/2005/11/29/17215/709).

Kiesler, Sara. 1997. Culture of the Internet. Mahwah, NJ: Lawrence Erlbaum Associates, Inc.

Kollock, Peter. 1999. "The Economies of Online Cooperation: Gifts and Public Goods in Cyberspace." Pp. 220-239 in Communities in Cyberspace, edited by M. A. Smith and P. Kollock. New York, NY: Routledge.

Kollock, Peter and Marc Smith. 1994. "Managing the Virtual Commons: Cooperation and Conflict in Computer Communities." University of California, Los Angeles, Los Angeles, CA. Retrieved November 30, 2006 (http:/www.sscnet.ucla.edu/soc/csoc/papers/virtcomm/Virtcomm.htm)

Lampe, Cliff and Paul Resnick. 2004. "Slash(Dot) and Burn: Distributed Moderation in a Large Online Conversation Space." CHI Letters 6:543-550.

Lampe, Cliff and Erik Johnson. 2005. "Follow the Slash(Dot): Effects of Feedback on New Members in an Online Community." Pp. 11-20 in International ACM 
SIGGROUP Conference on Supporting Group Work. Sanibel Island, FL: ACM Press.

Littlejohn, Stephen W. and Kathy Domenici. 2001. Engaging Communication in Conflict: Systemic Practice. Thousand Oaks, CA: Sage Publications.

Lyons, Gay Henry 2002. "Civic Engagement in the Cyberspace Era: A Study of a Local Cybergroup." Doctor of Philosophy Thesis, Political Science, University of Tennessee, Knoxville, Knoxville, TN.

Madden, Mary and Susannah Fox. 2006. "Riding the Waves Of "Web 2.0"." Pew Internet and American Life Project, Washington, DC.

McKenna, Katelyn Y. A. and Amie S. Green. 2002. "Virtual Group Dynamics." Group Dynamics: Theory, Research, and Practice 6:116-127.

McKenna, Katelyn Y. A. and J. A. Bargh. 1999. "Causes and Consequences of Social Interaction on the Internet: A Conceptual Framework." Media Psychology 1:249-269.

McKenna, Katelyn Y. A., Amie S. Green, and Marci E. J. Gleason. 2002. "Relationship Formation on the Internet: What's the Big Attraction?." Journal of Social Issues 58:9-31.

Powazek, Derek M. 2002. Design for Community: The Art of Connecting Real People in Virtual Places. Indianapolis, IN: New Riders.

Preece, Jenny. 2001. "Sociability and Usability in Online Communities: Determining and Measuring Success." Behaviour \& Information Technology 20:347-356.

Rafaeli, Sheizaf and Fay Sudweeks. 1997. "Networked Interactivity." Journal of Computer Mediated Communication 2. Retrieved November 30, 2006 (http://jcmc.indiana.edu/vol2/issue4/rafaeli.sudweeks.html).

Rainie, Lee. 2005. "The State of Blogging." Pew Research Center: Pew Internet and American Life Project.

Rainie, Lee and John B. Horrigan. 2005. "A Decade of Adoption: How the Internet Has Woven Itself into American Life." Pew Internet and American Life Project, Washington, DC.

Reid, Elizabeth. 1999. "Hierarchy and Power: Social Control in Cyberspace." Pp. 107-133 in Communities in Cyberspace, edited by M. A. Smith and P. Kollock. New York, NY: Routledge.

Rheingold, Howard. 2000. The Virtual Community: Homesteading on the Electronic Frontier, vol. 2003. Cambridge, MA: MIT Press.

Riva, Giuseppe. 2002. "Sociocognitive Psychology of Computer-Mediated Communication: The Present and Future of Technology-Based Interactions." Cyberpsychology and Behavior 5:581-598. 
Rourke, Liam, Terry Anderson, D.R. Garrison, and Walter Archer. 2001.

"Methodological Issues in the Content Analysis of Computer Conference Transcripts." International Journal of Artificial Education 12.

Serfaty, Viviane. 2002. "Forms and Functions of Conflict in Online Communities." Cercles 5:183-197.

Shea, Virginia. 1994. Netiquette. San Francisco, CA Albion Books.

Sitemeter. 2006, "Counter and Statistics Tracker for the Daily Kos", Retrieved

March 6, 2006, (http://www.sitemeter.com/?a=stats\&s=sm8dailykos\&r=0).

Suler, John. 2004. "The Online Disinhibition Effect." Cyberpsychology and behavior 7:321-326.

Sunstein, Cass. 2001. Republic. Com. Princeton, NJ: Princeton University Press.

Turkel, Sherry. 1995. Life on the Screen: Identity in the Age of the Internet. New York, NY: Touchstone Books.

Wallace, Patricia. 1999. The Psychology of the Internet. New York, NY: Cambridge University Press.

Wilmot, William W. and Joyce L. Hocker. 1998. Interpersonal Conflict. Boston, MA: McGraw-Hill.

Zafeiriou, Georgia. 2003. "Managing Conflict and Reaching Consensus in TextBased Computer Conferencing: The Students' Perspective." Education for Information 21:97-111. 


\section{APPENDIX A - DIARIES AND CONVERSATION THREADS}

The original diaries which formed the dataset for this research, and the twelve conversation threads which were analyzed, can be found online at this address: http://www.roundthree.net/soma_thesis_dailykos/

The original diaries are still "live" on the Daily Kos site and can be accessed by anyone with an Internet connection at the time of this writing. The conversation threads and the originating diaries are hosted on a privately hosted website. In the event that the privately hosted website goes offline, the author will respond affirmatively to any requests for the conversation threads which were analyzed in this research. The conversation threads are:

\begin{tabular}{|l|l|l|l|l|l|l|}
\hline Thread & Title & $\begin{array}{l}\text { Total \# } \\
\text { authors }\end{array}$ & $\begin{array}{l}\text { Total \# } \\
\text { comments }\end{array}$ & Thread type & $\begin{array}{l}\text { \# rated } \\
\text { comments } \\
\text { /ratings }\end{array}$ & $\begin{array}{l}\text { \# Authors (a) } \\
\text { \&\# } \\
\text { Comments (c) } \\
\text { written }\end{array}$ \\
\hline $1(2)$ & $\begin{array}{l}\text { Don't take } \\
\text { this bait }\end{array}$ & 10 & 20 & $\begin{array}{l}\text { Benign conflict, } \\
\text { and discussion of } \\
\text { situation }\end{array}$ & $3 / 16$ & $\begin{array}{l}1 \mathrm{a}-4 \mathrm{c} \\
2 \mathrm{a}-3 \mathrm{c} \\
3 \mathrm{a}-2 \mathrm{c} \\
4 \mathrm{a}-\mathrm{lc}\end{array}$ \\
\hline $2(6)$ & $\begin{array}{l}\text { The Final } \\
\text { Chapter of } \\
\text { Delaware }\end{array}$ & 10 & 19 & $\begin{array}{l}\text { \#1 informational } \\
\text { Dem no response } \\
\text { \#3 benign } \\
\text { conflict }\end{array}$ & $2 / 11$ & $\begin{array}{l}1 \mathrm{a}-4 \mathrm{c} \\
2 \mathrm{a}-3 \mathrm{c} \\
2 \mathrm{a}-2 \mathrm{c} \\
5 \mathrm{a}-1 \mathrm{c}\end{array}$ \\
\hline $3(17)$ & $\begin{array}{l}\text { DD, resident } \\
\text { piethrower }\end{array}$ & 4 & 10 & $\begin{array}{l}\text { Started as } \\
\text { conversation, } \\
\text { then became } \\
\text { Conflict w/DD } \\
\text { antagonizing, feel } \\
\text { free to leave }\end{array}$ & 0 & $\begin{array}{l}1 \mathrm{a}-4 \mathrm{c} \\
3 \mathrm{a}-2 \mathrm{c}\end{array}$ \\
\hline $4(26)$ & $\begin{array}{l}\text { Thanks for } \\
\text { Nothing DD }\end{array}$ & 12 & 16 & $\begin{array}{l}\text { Conflict - } \\
\text { Armando-delete. } \\
\text { \& discussion of } \\
\text { wider conflict }\end{array}$ & $5 / 27$ & $\begin{array}{l}1 \mathrm{a}-3 \mathrm{c} \\
2 \mathrm{a}-2 \mathrm{c} \\
9 \mathrm{a}-1 \mathrm{c}\end{array}$ \\
\hline $5(28)$ & $\begin{array}{l}\text { "petty little } \\
\text { sensitivities" }\end{array}$ & 4 & 6 & Conflict & $1 / 2$ & $\begin{array}{l}2 \mathrm{a}-2 \mathrm{c} \\
2 \mathrm{a}-1 \mathrm{c}\end{array}$ \\
\hline
\end{tabular}




\begin{tabular}{|l|l|l|l|l|l|l|}
\hline $6(31)$ & $\begin{array}{l}\text { What I really } \\
\text { want to know } \\
\text { is }\end{array}$ & 7 & 18 & $\begin{array}{l}\text { Conflict - started } \\
\text { with question, got } \\
\text { joke response, } \\
\text { escalation } \\
\text { followed. }\end{array}$ & $3 / 15$ & $\begin{array}{l}1 \mathrm{a}-6 \mathrm{c} \\
2 \mathrm{a}-4 \mathrm{c} \\
4 \mathrm{a}-1 \mathrm{c}\end{array}$ \\
& & & & \\
\hline
\end{tabular}

Table 1 - Diary 10 - It is an Ad. Nothing More. Delaware Dem

\begin{tabular}{|c|c|c|c|c|c|c|}
\hline Thread & Title & $\begin{array}{l}\text { Total \# } \\
\text { of } \\
\text { authors }\end{array}$ & $\begin{array}{l}\text { Total \# } \\
\text { comments }\end{array}$ & Thread Type & $\begin{array}{l}\text { \# rated } \\
\text { comments } \\
\text { / ratings }\end{array}$ & $\begin{array}{l}\text { \# Authors } \\
\text { (a) \&\# } \\
\text { Comments } \\
\text { (c) written }\end{array}$ \\
\hline $1(1)$ & $\begin{array}{l}\text { Don't } \\
\text { Worry }\end{array}$ & 17 & 30 & Conflict & $\begin{array}{l}10 / 61 \\
\text { ratings }\end{array}$ & $\begin{array}{l}1 a-5 c \\
2 a-3 c \\
\text { each } \\
7 a-2 c \\
\text { each } \\
9 a-1 c \\
\text { each }\end{array}$ \\
\hline $2(6)$ & $\begin{array}{l}2 \text { main } \\
\text { responses }\end{array}$ & 4 & 6 & $\begin{array}{l}\text { Conversation } \\
\text { (explanation of } \\
\text { GBCW), but started } \\
\text { with description of } \\
\text { problem }\end{array}$ & $\begin{array}{l}2 / 14(1 \mathrm{st} \\
\text { comment } \\
\text { got } 12 \\
\text { ratings) }\end{array}$ & $\begin{array}{l}2 a-2 c \\
2 a-1 c\end{array}$ \\
\hline $3(7)$ & $\begin{array}{l}\text { Um... } \\
\text { MSOC }\end{array}$ & 23 & 44 & $\begin{array}{l}\text { Conflict - Hink's } \\
\text { apology. Lots of } \\
\text { discussion }\end{array}$ & $12 / 37$ & $\begin{array}{l}1 a-8 c \\
1 a-6 c \\
1 a-3 c \\
7 a-2 c \\
13 a-1 c\end{array}$ \\
\hline $4(8)$ & tis & 2 & 4 & Conversation & 12 & $2 a-2 c$ \\
\hline $5(13)$ & $\begin{array}{l}\text { I find it } \\
\text { somewhat } \\
\text { ironic that } \\
\text { so many... }\end{array}$ & 2 & 5 & $\begin{array}{l}\text { True conflict with } \\
\text { apology. No need for } \\
\text { extra conversants. }\end{array}$ & $1 / 3$ & $\begin{array}{l}1 a-3 c \\
1 a-2 c\end{array}$ \\
\hline $6(15)$ & $\begin{array}{l}\text { I don't } \\
\text { think Page } \\
\text { has left us }\end{array}$ & 4 & 7 & $\begin{array}{l}\text { Conflict with } \\
\text { apology. }\end{array}$ & $2 / 6$ & $\begin{array}{l}1 a-3 c \\
1 a-2 c \\
2 a-1 c\end{array}$ \\
\hline
\end{tabular}

Table 2 - Diary 11 - Is Plutonium Page Gone Too? Paradox 


\section{APPENDIX B - ADDITIONAL BACKGROUND ON THE DAILY KOS SITE \\ Site History}

Daily Kos (so named because "Kos" was the owner's nickname when he served in the U.S. Army) is owned by Markos Moulitsas Zúniga, and has been in existence since May of 2002. As of December 2005, Daily Kos was the most highly trafficked political blog (522708 visits/day) as calculated by the Truth Laid Bear Blogging Ecosystem.

User registration is required for those who would like to post diaries or comments, recommend diaries, or rate comments on the site, although read-only access to the site is available to anyone. It is free to register on the site, and there are no formal posting requirements or limitations, excepting a one-week probationary period limiting the user's ability to post a diary. While previous iterations of the site (and many other political blogs) do not employ this registration barrier to commenting, Daily Kos moved to this format in October of 2003. The reasons for this change included the fact that the anonymous comment format incurred several cases of inundation by advertising spammers, trolling, mistaken identity, and even identity theft. As well, users could only read comments chronologically, and could not reply directly to a comment which occurred earlier in the thread. These software limitations hindered the interactivity of the site and the substance of the commentary. This does not mean that users did not quote other users to designate to whom or to what specifically they were replying, but the process required an extra step that not every user chose (or was technologically astute enough) to employ. Later research 
might compare the substance of the commentary and interactivity of the comments in the earlier comments threads and within the current system.

Moulitsas began taking in advertising revenue in late 2003 to support the site's operational costs. Members who wish to view an ad-free version of the site may purchase a site subscription for the cost of $\$ 4 /$ month, $\$ 40 /$ year, or $\$ 100$ for a lifetime subscription. Subscribers receive no additional content or access privileges for their subscription.

\section{Format}

The study community is a combination of a blog and a Usenet group. A seminal article, called a Diary, is posted on a topic. Community members are invited to post responses, or comments, about the article.

The Kos community is primarily self-regulating, with the posting members taking an active interest in the health and wellbeing of the community. During the week of my study period, approximately 5,000 individuals posted diaries and comments, both within and outside of the Pie Fight articles. It is not known how many of the registered members read the site without posting on it, but the overall site statistics run on the order of over one million visits a week. These site statistics include both registered and non-registered readers.

There is an active encyclopedia of the site, called the dKosopedia, a play on the word encyclopedia, which includes information about the site and various political concerns. 


\section{Membership}

As noted above, anyone at all can view the site or its comments, membership privileges include the ability to comment on posts, create diaries, and rate other members' posts for quality. The Daily Kos forum is similar to earlier political forums in that registration is open to anyone with an email address and there are certainly people who post with the desire to "win" more than the desire for earnest, open political discussion. However, given that Daily Kos also enables its users to screen out undesirable content (via the comment-rating system discussed below) the posts which appear are relevant, interesting, and serve to inspire discussion among members, rather than posts which contain personal attacks or put-downs that typically serve to shut down discussion.

As of November 4, 2005, there were 70,555 individual user names registered on the site, although the actual posting figure is closer to 6,000 in any given week in 2005. There is no indication anywhere on the site of how many people are paid subscribers.

\section{Site Layout}

\section{The Front Page - Stories and Front Page Authors}

The Daily Kos site has various areas where readers of the site can read originating articles. Originating articles on the front page of the site are known as stories. These stories are written by the site owner plus people within the Daily Kos community who have been given the privilege of front-page posting status by the site 
owner. In some cases, the community has voted on who should be given front page posting status. As described below, diaries may be "promoted" to the front page by any of the front page authors. The author of the diary retains their diary author status, but their site status is increased by the promotion, since their diary will likely be seen by the same number of people who view the front page stories.

\section{Open Threads Stories}

Interspersed with the Stories on the front page are Open Threads, wherein comments of any type are permitted. There are two types of Open Threads: those that are auto-generated (under the open thread author) and those that are typically written by Moulitsas or another front page author. Sometimes there are themes of the open threads, but they are primarily designed for community members to comment and/or discuss issues that have not been covered within a recent Story, or are not otherwise deserving of front page treatment. In many cases, the open threads are where the community aspect of the Daily Kos shines through. It is here that inside-jokes are shared (or explained), community building occurs, where references to other diaries that didn't make the front page or the recommended list are made, and where community members promote their own diaries.

There is also a "Cheers and Jeers" story which functions in the same way as an open thread, but is not considered as such. The official cheers and jeers author, Bill in Portland ME, has been accorded "unofficial" front page author status in a tacit 
admission of the value such a generic but traditional practice offers to the community.

To the right of the front page stories are the advertisements. These are typically combinations of text and images, and are only hidden if a community member has purchased a subscription to the site (discussed above).

\section{Diaries}

Diaries are originating articles which are written by community members who do not have front-page posting status. Diaries which are determined to be worthy of community-wide exposure are "promoted" to the front page of the Daily Kos site, either by Moulitsas or by one of the other five front page authors. As well, there is a dynamically created list of user-recommended diaries on the front page. The title of the diary, the diary author, and the number of comments it has received are all that is visible on this list, but the site viewer can click on the title to see the entire diary and comments. Registered users can select the number of "most recommended diaries" that they would like to view in the user preferences area. The default number of recommended diaries on view is 10 .

There is also a dynamically created list of recently posted diaries - the user can choose how many recently posted diaries they would like to see on the front page. Alternately, there is a link to the main diaries page where all diaries are posted. Since the Daily Kos community has almost 80,000 users, it would be impossible to 
keep up with all of the diaries that are written - approximately 200 diaries are written per day.

\section{Commenting}

After an article or diary is written, community members can extend the dialogue by commenting on the diary, and/or on a previous user's comments on the diary. This results in several threads of conversation, with the initiating post typically being in response to the original diary (Comment Code $\mathrm{A}$ ) and subsequent posts being in response to Comment Code $\mathrm{A}$.

A "tip jar" comment is often posted by the diarist as a means of increasing his/her Mojo (see below). Since diary recommendations are not included in Mojo calculations, this is often the only means by which diarist's contributions are measured or rewarded. The tip jar practice is resisted by some diarists, but is predominantly an understood and supported convention of the site.

\section{Rating / Mojo / Trusted User Privileges}

As mentioned in the main document, ratings on the Daily Kos site are a means of participating in the dialog without having to take the time to write. Comments are rated from $0-4$ in order of increasing "value" to the community, although 0 is a rating only trusted users may give, and which hides the comment from view.

In the interests of giving back to the community, and perhaps to avoid the result of hiding comments, or else because those who do not have the power to give 
zeroes would still like to have input on the conversation, a convention has arisen on Daily Kos wherein a user will post a recipe as a comment to a post that they would have rated as Zero. This is an interesting and useful modification of the means by which a community member can admonish another, and is noteworthy because the posting of a recipe likely requires much more effort than merely rating a post or user with a zero.

Mojo is an idea borrowed from the Slashdot community which ostensibly helps to keep the comments on the site as "high quality as possible." Any user can rate any comment except their own, and the ratings are intended to reward users who craft insightful or informative comments while weeding out the "trolls" who might invade and destroy the community. Ratings are not supposed to indicate agreement or disagreement with a particular commenter or point of view, but there is no formal prohibition against a user only choosing to rate comments based on this criteria. Raters who go on "sprees" and negatively rate (a practice known as downrating) multiple comments are usually noticed and sanctioned by the community. Oftentimes, a commenter who receives a bad rating will post a second comment within the thread to demand that the person who gave the rating give an explanation. Occasionally, retaliatory downrating occurs, but if so, it is usually noted within the comment thread. Concern about ratings is typically seen as a counterproductive to the conversation, and is looked down upon by the community.

The reason that comment ratings are so important is that a user's comment ratings are combined into a weighted average which is results in a Mojo value. The 
Mojo value is the expected rating of a user's subsequent comments. After a user has posted a sufficient number of comments, and has also obtained a Mojo value which is higher than a certain minimum, that user attains Trusted User status. A Trusted User is able to rate comments below the normal minimum rating (e.g. they may rate comments as $0-4$, rather than 1-4). This privilege allows a trusted user to join in the policing of Daily Kos - if enough of a user's comments are rated below one, then that user is "untrusted." There are no specific sanctions to this rating, although comments which have an average rating of below 1 are hidden from the view of regular daily Kos readers.

\section{Becoming a Trusted User}

While the means by which a regular user becomes a trusted user are very generally explained, the actual calculations are not made transparent to the community members. What is known is that a user who makes either comments or diaries that are favorably rated by the community eventually becomes elevated to this status. Trusted Users have three specific privileges that Regular Users do not: they can anonymously give comments a zero (troll) rating, which might end up removing the comment from view; they can see all hidden, troll-rated comments; and they can see who has given a comment a zero rating.

There is no transparency in the community about how many users are actually Trusted Users, nor is a user formally informed that they have attained this status. When trusted user status is discussed in comments, most trusted users note 
that they realized they'd been elevated because their ratings scheme had changed from 1-4 to 0-4 (with zero being a troll rating - if a comment gets an average of $<1$, then it is hidden from view of the regular community). Markos himself, when asked how the calculations are performed, and by what means a user is accorded this status, has simply stated "Don't worry about it - it's not a big deal," However, since the Trusted Users are able to hide certain posts from the typical user's view without either detection or accountability, the Trusted User likely has a greater influence on the community than is immediately apparent.

\section{Tags}

As of October 2005, author defined tags/keywords are attached to each diary that is posted, and site users are able to search for diaries that are tagged with specific keywords. Although each community member may create their own tag, and many do, there has been a great deal of discussion about the rules for tagging posts. For now, Trusted Users are empowered to remove inappropriate tags from diary posts if they so choose, and authors are asked to use preexisting, somewhat generic tags rather than create idiosyncratic or "clever" tags that aren't as useful in terms of narrowing a search for a specific diary subject. 